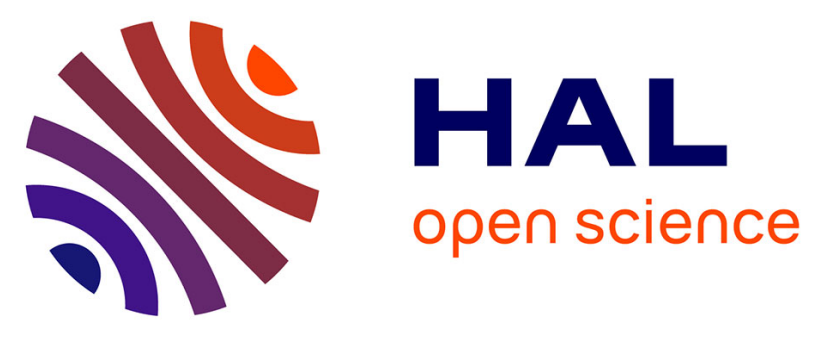

\title{
MAVEN and MEX multi-instrument study of the dayside of the Martian induced magnetospheric structure revealed by pressure analyses
}

Mika K. G. Holmberg, Nicolas Andre, Philippe Garnier, Ronan Modolo, Laila Andersson, Jasper S. Halekas, Christian Mazelle, Morgane Steckiewicz, Vincent Genot, Andrei Fedorov, et al.

\section{To cite this version:}

Mika K. G. Holmberg, Nicolas Andre, Philippe Garnier, Ronan Modolo, Laila Andersson, et al.. MAVEN and MEX multi-instrument study of the dayside of the Martian induced magnetospheric structure revealed by pressure analyses. Journal of Geophysical Research Space Physics, 2019, 124 (11), pp.8564-8589. 10.1029/2019JA026954 . insu-02318363

\section{HAL Id: insu-02318363 \\ https://hal-insu.archives-ouvertes.fr/insu-02318363}

Submitted on 29 Mar 2021

HAL is a multi-disciplinary open access archive for the deposit and dissemination of scientific research documents, whether they are published or not. The documents may come from teaching and research institutions in France or abroad, or from public or private research centers.
L'archive ouverte pluridisciplinaire HAL, est destinée au dépôt et à la diffusion de documents scientifiques de niveau recherche, publiés ou non, émanant des établissements d'enseignement et de recherche français ou étrangers, des laboratoires publics ou privés. 


\section{JGR Space Physics}

\section{RESEARCH ARTICLE \\ 10.1029/2019JA026954 \\ Key Points: \\ MAVEN and MEX Multi-instrument Study of the Dayside of the Martian Induced Magnetospheric Structure Revealed by Pressure Analyses}

- This article presents an overview of the Martian dayside magnetospheric structure based on the dominant pressure terms

- Typical altitudes of the pressure balance boundaries, the PEB, ICB, and IMB are provided

- We show that earlier defined boundaries are not a sufficient characterization of the ionosphere/magnetosheath transition region

Correspondence to: M. K. G. Holmberg, mika.holmberg@esa.int

Citation:

Holmberg, M. K. G., André, N., Garnier, P., Modolo, R., Andersson, L., Halekas, J., et al. (2019). MAVEN and MEX multi-instrument study of the dayside of the Martian induced magnetospheric structure revealed by pressure analyses. Journal of Geophysical Research: Space Physics, 124, 8564-8589. https://doi.org/10.1029/ 2019JA026954

Received 18 MAY 2019 Accepted 23 SEP 2019 Accepted article online 16 OCT 2019 Published online 8 NOV 2019

Corrected 11 FEB 2020

This article was corrected on 11 FEB 2020. See the end of the full text for details.

(C)2019. American Geophysical Union. All Rights Reserved.

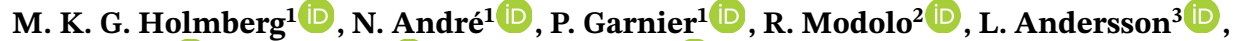 \\ J. Halekas ${ }^{4}$ (D) C. Mazelle ${ }^{1}$ (D) M. Steckiewicz ${ }^{1}$ (D) , V. Génot ${ }^{1}$, A. Fedorov ${ }^{1}$, S. Barabash ${ }^{5}$, \\ and D. L. Mitchell ${ }^{6}$ iD \\ ${ }^{1}$ IRAP, University of Toulouse, CNRS, UPS, CNES, Toulouse, France, ${ }^{2}$ LATMOS/IPSL, UVSQ Université Paris-Saclay, \\ UPMC University Paris CNRS, Guyancourt, France, ${ }^{3}$ LASP, University of Colorado Boulder, Boulder, CO, USA, \\ ${ }^{4}$ Department of Physics and Astronomy, University of Iowa, Iowa City, IA, USA, ${ }^{5}$ Swedish Institute of Space Physics, \\ Kiruna, Sweden, ${ }^{6}$ Space Sciences Laboratory, University of California, Berkeley, CA, USA
}

\begin{abstract}
A combination of statistical studies and 18 case studies have been used to investigate the structure of the induced Martian magnetosphere. The different plasma and magnetic pressure forces on the dayside of the induced magnetosphere of Mars have been studied using 3.5 years of Mars Atmosphere and Volatile Evolution (MAVEN) and Mars Express (MEX) observations. We present estimates of typical values for the dominant pressure terms, that is, the thermal pressures of the ionosphere and the magnetosheath, the magnetic pressure of the magnetic pile-up region, and the solar wind dynamic pressure. For 18 typical orbits the altitudes and relative distances of the pressure balance boundaries, the photoelectron boundary, the ion composition boundary, and the induced magnetosphere boundary are estimated. The magnetic pile-up boundary is discussed but not further studied since earlier characterizations of the magnetic pile-up boundary do not agree with our results. This study focuses on the transition region between the ionosphere and the magnetosheath on the dayside of Mars. We show that earlier definitions of the photoelectron boundary, ion composition boundary, and induced magnetosphere boundary do not characterize the transition region well, mainly because each boundary is based on measurements from only one or two instruments. In order to characterize the transition region correctly, changes in magnetic field strength and fluctuations, dominant ion species, electron and ion densities and energy distributions need to be considered. This article confirms a complex interaction between Mars and the solar wind and can explain why previous studies have had difficulties to describe the force balance.
\end{abstract}

\section{Introduction}

Unlike Earth, Mars do not possess a global magnetic field. Due to this, the interaction with the solar wind occurs directly with the planetary ionosphere and upper atmosphere, in a manner similar to the solar wind interaction with Venus or comets; that is, an induced magnetosphere is created. The interaction, however, is further complicated by the presence of strong crustal magnetic fields that are believed to be remnants of a global magnetic field that Mars once had, before the dynamo stopped (Acuña et al., 1998). The crustal magnetic fields create mini-magnetospheres that trap particles and alter the local structure of the induced magnetosphere. The Martian induced magnetosphere is hence a complicated structure that requires both good spatial and temporal coverage for an accurate characterization. For reviews of the Martian induced magnetosphere see, for example, Nagy et al. (2004), Mazelle et al. (2004), or Russell (2007).

Historically, a large variety of names have been suggested for the boundaries found to separate the different regions of the dayside magnetosphere of Mars, partly due to the limited plasma and magnetic field instrumentation on the early Mars missions. The first studied boundary was the bow shock of the planet, which has been studied in situ since the earliest missions to Mars, such as the Mariner 4 flyby in 1965 and the Mars 2, 3, and 5 orbiters in 1971-1974. At that time, however, the characteristics of the plasma environment within the bow shock were still unknown and the question if Mars had a global magnetic field or not was still not resolved. 
Within the bow shock, measurements by the Phobos 2 spacecraft in 1989 found a boundary characterized both by changes in the magnetic field and the ion composition. This boundary was named planetopause by Riedler et al. (1989) and Grard et al. (1989) and magnetopause by Lundin et al. (1989), Rosenbauer et al. (1989), and Shutte et al. (1989). Later missions provided more detailed analyses of the Martian dayside magnetosphere, and several plasma boundaries were identified, replacing the previously used planetopause and magnetopause of Mars. The Mars Global Surveyor (MGS) spacecraft, 1997-2006, detected the magnetic pile-up boundary (MPB), which separates the magnetosheath and the magnetic pileup region (MPR) (Acuña et al., 1998). This boundary was described as marked by a strong increase in the magnetic field strength, reduced magnetic field fluctuations, and decreased suprathermal electron fluxes, when moving from the magnetosheath to the pile-up region (Acuña et al., 1998; Vignes et al., 2000; Bertucci et al., 2003). Another similar boundary found in the same altitude range was called the induced magnetosphere boundary (IMB) (e.g., Lundin et al., 2004); it was defined as the boundary between the solar wind and the plasma of planetary origin. The name IMB was used instead of MPB since Mars Express (MEX) did not carry a magnetometer and magnetic field measurements, essential for studying the MPB, were not available.

A boundary referred to as the ion composition boundary (ICB) was also detected by Phobos 2 (Breus et al., 1991). The ICB was characterized by a sharp drop in proton fluxes over a distance of around $100 \mathrm{~km}$ and an increase in planetary ions, mainly $\mathrm{O}^{+}$ions, with around one order of magnitude (Breus et al., 1991). It was argued that this boundary was identified at the same distance as the planetopause or magnetopause but that the ICB was a more accurate nomenclature, since the detected boundary was not a "pause" but a more smooth transition region (Breus et al., 1991). However, later studies, including this study, show that the change in ion composition is not found at the same distance as the changes in magnetic field strength and fluctuations. The ICB was further studied by Bößwetter et al. (2004) who used a 3-D hybrid code, where the ions was treated kinetically, to show that an ICB will be obtained because the pressure gradient of the electrons at the ICB will generate a strong electric field that will deflect the solar wind ions. Bößwetter et al. (2004) showed that an increased ionospheric electron density and/or temperature will result in an increased ICB altitude.

A statistical study of the altitudes of the bow shock and the MPB was presented by Vignes et al. (2000). They used MGS magnetometer investigation (MAG) data from 290 orbits to obtain a fit to the geometrical characteristics, assuming the shape of the boundaries as conic sections rotationally symmetric around an aberrated axis that is in the opposite direction of the mean solar wind flow direction. They obtained a subsolar stand-off distance for the bow shock of $1.64 \pm 0.08$ Mars radii (where $1 R_{M}=3,396.2 \mathrm{~km}$ ) and $1.29 \pm 0.04 R_{M}$ for the MPB. A follow-up study was presented by Trotignon et al. (2006) who combined Phobos 2 and MGS measurements for larger distances to obtain stand-off distances of $1.63 \pm 0.01 R_{M}$ and $1.25 \pm 0.03 R_{M}$ for the bow shock and MPB, respectively. The altitude of the bow shock and the MPB as a function of solar wind dynamic pressure, crustal magnetic fields, solar extreme ultraviolet (EUV) flux, and the interplanetary magnetic field (IMF) direction were studied by Edberg et al. (2009). They used around 3 years of MGS and MEX, 2003 to present, measurements to show that all of the listed parameters have a significant impact on the altitudes and shapes of the studied boundaries.

Measurements by the MGS Electron Reflectometer (ER) also revealed a boundary with a median altitude of $380 \mathrm{~km}\left(1.11 R_{M}\right)$ characterized by a strong change in the electron spectra with an abrupt drop in electron fluxes greater than $\sim 100 \mathrm{eV}$ (Mitchell et al., 2000). Above the boundary the electron energy spectra show the shocked sheath electrons and below the boundary the spectra showed a broad feature in the energy range $20-50 \mathrm{eV}$, identified as photoelectrons of ionospheric origin (Mitchell et al., 2000). The finer energy resolution of the MEX Analyzer of Space Plasma and Energetic Atoms (ASPERA-3) Electron Spectrometer was able to resolve the two expected main photoelectron peaks in the energy range 20-30 eV (e.g., Mantas and Hanson, 1979) and the boundary was referred to as the photoelectron boundary (PEB) (Lundin et al., 2004). Garnier et al., (2017) used Mars Atmosphere and Volatile Evolution (MAVEN), 2014 to present, measurements from almost 1,700 boundary crossings to study the PEB further. They showed that the PEB is an approximately circular boundary with an average altitude of $573 \mathrm{~km}\left(1.17 R_{M}\right)$. However, the PEB is also shown to be highly variable, varying from $186 \mathrm{~km}\left(1.05 R_{M}\right)$ to $1,931 \mathrm{~km}\left(1.57 R_{M}\right)$, mainly depending on the crustal field strength, the solar wind dynamic pressure, and the local time (LT) (Garnier et al., 2017). 
Dubinin et al. (2008) studied the structure and dynamics of the solar wind/ionosphere interface using MEX measurements from four orbits in July 2007. This was the first attempt to compare all the different pressure terms on the dayside of the Martian magnetosphere. They concluded that the solar wind/ionosphere interface is very dynamic and that the structure of the pressure balance boundary between the MPR and the magnetosheath varies considerably. The MPR is the region just below the magnetosheath and is defined as a region of increased magnetic field strength, commonly with more than a factor of 5, compared to the field strength in the magnetosheath. Dubinin et al. (2008) also showed that the transition from the IMB to the PEB is accompanied by an increase in plasma density. An extended study, which also included MEX observations of the nightside magnetosphere, was presented by Dubinin et al. (2008). They showed that the process of the IMB formation is governed by the solar wind and that the solar wind/ionosphere interface only occasionally could be described with the previously stated definition of the MPB.

Xu et al. (2016) used a Block-Adaptive Tree Solar wind Roe-type Upwind Scheme Mars Multifluid magnetohydrodynamic model to study the three-dimensional shape and structure of the pressure boundaries and the ICB. They found that the thickness of the boundaries are 0.05-0.1 $R_{M}(170-340 \mathrm{~km})$ near the subsolar region and 1-1.5 $R_{M}(3,396-5,094 \mathrm{~km})$ in the magnetotail. Matsunaga et al. (2017) presented a statistical study of the relationships between the IMB, ICB, and the pressure balance boundaries using MAVEN measurements recorded between November 2014 and October 2015. They showed that the pressure balance boundary between the MPR and the magnetosheath tend to be located at higher altitude than the IMB and the ICB, that there is a northern southern asymmetry in the boundary altitudes on the nightside, and that the solar wind dynamic pressure is the main driver for all the studied boundaries. Halekas et al. (2018) presented a detailed study of the ICB location, characteristics, and variability. They used 9,613 ICB crossings, obtained from MAVEN Suprathermal and Thermal Ion Composition (STATIC) measurements, to show that the thickness, altitude, and velocity shear of the ICB depends on the direction of the solar wind motional electric field. They also showed that the ICB location varies with solar wind ram pressure and crustal magnetic field strength.

The variety of names used for the boundary between the ionosphere and the magnetosheath can be explained by several limiting factors. For example, many studies were biased by the authors preferred instrument, based on only one or two measurement types, and restricted by the limited plasma and/or magnetic field instrumentations of the various missions. The unsettled terminology concerning the induced magnetosphere of Mars is further discussed by Espley (2018). In this study we will investigate the various boundaries, using the earlier definitions, to estimate their average altitudes and their relative distances, and discuss their importance as a representation of the boundary between the Martian ionosphere and magnetosheath.

In this study we take advantage of the simultaneous measurements by MAVEN and MEX to study the structure of, and the plasma boundaries found in, the dayside of the induced magnetosphere of Mars. The MAVEN spacecraft, founded and operated by NASA, has been orbiting Mars since September 2014. Its main objectives are to study the upper atmosphere of Mars and its interaction with the solar wind, with a special focus on atmospheric escape (Jakosky et al., 2015). MAVEN's comprehensive plasma package, including both several particle analyzers and a magnetometer, makes it possible to study the Martian induced magnetosphere in more detail than ever before and to further investigate findings from earlier Mars missions. We also use measurements from the MEX spacecraft, which has been orbiting Mars since December 2003. MEX is founded and operated by the European Space Agency. Its main objectives are to search for signs of water, to study the compositions and structures of the surface and atmosphere, and to study the interaction between the solar wind and the upper atmosphere of Mars. For a comprehensive overview of the MEX mission see Chicarro et al. (2004). We use MEX data from the ASPERA-3 instrument to obtain the dynamic pressure in the solar wind.

\section{Observations}

MAVEN is orbiting Mars in an elliptical orbit with a periapsis between 150 and $200 \mathrm{~km}\left(1.04-1.06 R_{M}\right)$, apoapsis between 5,900 and $6,000 \mathrm{~km}\left(2.74-2.77 R_{M}\right)$, and an inclination of $75^{\circ}$. One orbit takes about $4.5 \mathrm{hr}$ to complete. Occasional deep-dip campaigns take the spacecraft down to $112-130 \mathrm{~km}\left(\sim 1.03 R_{M}\right)$. MEX has a near polar orbit with a periapsis between 245 and $365 \mathrm{~km}\left(1.07-1.11 R_{M}\right)$, apoapsis between 10,107 and $11,560 \mathrm{~km}\left(3.98-4.40 R_{M}\right)$, and an inclination of $85^{\circ}$. One orbit takes between 6.7 and $7.5 \mathrm{hr}$ to complete. 
For this study we use data from the MAVEN instruments that are listed below. The Langmuir Probe and Waves (LPW) instrument consists of two Langmuir probes that measure electron density from $\sim 100$ to $10^{6} \mathrm{~cm}^{-3}$ and temperature from 500 to 50,000 K (Andersson et al., 2015). The LPW measurements are used to obtain the plasma density and electron temperature in the Martian ionosphere. MAG consists of two identical fluxgate magnetometers that measures the magnetic field over dynamic ranges of \pm 512 or $\pm 65,536 \mathrm{nT}$ with resolutions 0.015 and $2 \mathrm{nT}$, respectively (Connerney et al., 2015). MAG is used to obtain the magnetic field strength measurements. The MAG measurements are used in combination with the LPW measurements to detect the lower pressure balance boundary. The STATIC instrument is a toroidal "top-hat" electrostatic analyzer that measures the ion composition and distribution function of ions with energies of 0.1 to $30 \mathrm{keV}$ (McFadden et al., 2015). The STATIC data are used to obtain the ion energy spectra and the ion mass spectra from which the ICB can be detected. The Solar Wind Electron Analyzer (SWEA) is an electrostatic analyzer that measures the energy and angular distributions of electrons with energies of 3 to $4,600 \mathrm{eV}$ (Mitchell et al., 2016). The SWEA measurements are used to obtain the electron energy spectra from which the PEB and the IMB can be detected. The Solar Wind Ion Analyzer (SWIA) is an electrostatic analyzer that measures density and velocity distributions of ions with energies of 5.1 to $26 \mathrm{keV}$ (Halekas et al., 2013). The SWIA data are used to obtain the ion density and velocity in the solar wind and the ion density and temperature in the magnetosheath. The SWIA data are used in combination with the MAG measurements in order to detect the upper pressure balance boundary.

We also use solar wind measurements from the MEX Analyzer of Space Plasmas and Energetic Atoms (ASPERA-3) Ion Mass Analyzer (IMA), which measures ions in the energy range 10 to $36 \mathrm{keV} / \mathrm{q}$ for the main ion components $\mathrm{H}^{+}, \mathrm{He}^{++}, \mathrm{He}^{+}, \mathrm{O}^{+}$, and the group of molecular ions 20 to $80 \mathrm{amu} / \mathrm{q}$ (Barabash et al., 2006). The MEX measurements are used to obtain solar wind density and velocity.

All of the instruments have their respective limitations and optimal measurements ranges, which are outlined in detail in the respective instrument paper. A number of limitations are important for this study, for example, that the SWIA onboard moment assumes $\mathrm{H}^{+}$to be the only present ion. In the solar wind this introduces an error of around 3\%, in the density moments, which will increase with decreasing altitude (Halekas et al., 2013). This assumption underestimates density and overestimates velocity whenever heavier ions are recorded. The field of view of SWIA is also a limiting factor, especially in the subsolar region, which is where the plasma flow is most deflected and decelerated (Halekas et al., 2017). This causes part of the distribution to fall outside of the field of view. SWIA should therefore be considered to provide a lower limit of the total ion density (Halekas et al., 2017).

To accurately determine the structure of the dayside of the Martian induced magnetosphere, this study takes advantage of both a statistical study, using 3 years of MAVEN data, and 18 case studies, using multipoint measurements. For the case study, a few orbit segments from the time interval between the MAVEN Mars orbit insertion, in September 2014, and the date 25 June 2017 were chosen. In order to reduce the limitation that the magnetospheric structure changes during the timescale over which the MAVEN spacecraft travels from the solar wind into the ionosphere, the MEX spacecraft is used for solar wind measurements. The orbits were chosen based on the conditions that both MAVEN and MEX were within solar zenith angle $(\mathrm{SZA})=60^{\circ}$, and best-quality data were provided by all necessary instruments; the MAVEN/LPW, MAG, SWEA, STATIC, and SWIA, and the MEX/ASPERA-3 instrument. These conditions limit the number of orbits to 29. Two additional conditions were also taken into consideration:

1. The crustal magnetic field has to be weak enough for the lower pressure balance boundary to be present. If the crustal magnetic field is strong, the magnetic pressure will always be dominant, and there will be no pressure boundary where the thermal pressure of the ionosphere is balanced with the magnetic pressure of the MPR. This condition removed five orbits.

2. Trajectories where two or more boundaries are undetectable are excluded. For example, orbits where MAVEN is crossing the lower pressure balance boundary at SZA $>60^{\circ}$ are excluded. This condition removed six orbits.

After the above conditions have been implemented, 18 orbit segments remain. The dates and times of the 18 orbit segments are listed in Table 2 column 1 . The 18 orbit segments will be studied in detail to obtain the altitudes of the upper and lower pressure balance boundaries, the PEB, ICB, and IMB. In section 3.2 we compare the 18 orbit segments with statistical studies of the various pressure terms in order to make sure that they represents the system well. 


\section{Results and Discussion}

To reveal the undisturbed structure of the dayside of the induced Martian magnetosphere, we study both the dominant pressure terms for the different regions from the ionosphere out to the solar wind and the altitudes of the various boundaries, such as the PEB, ICB, and IMB. The preferred notation in this study is IMB, instead of MPB, for the boundary that is characterized by a drop in suprathermal electron fluxes and a decrease in magnetic field fluctuations. This choice is due to the fact that the increase in the magnetic field strength, which is indicating the beginning of the MPR, often occurs hundred to several hundreds of $\mathrm{km}$, along the spacecraft orbit, above the detected decrease in suprathermal electron energy fluxes and magnetic field fluctuations. To identify the boundary as the MPB would therefore not be correct, since it is located at various altitudes within the MPR.

The different pressure terms are estimated using the following equations. The thermal pressure of the ionosphere $P_{t h, i}$ is estimated from the LPW electron density $n_{e}$ and temperature $T_{e}$, assuming quasineutrality $n_{e} \approx n_{i}$ and $T_{i} \approx T_{e} . P_{t h, i}$ is obtained using

$$
P_{t h, i}=2 n_{e} k_{B} T_{e}
$$

where $k_{B}$ is the Boltzmann constant. The thermal pressure of the magnetosheath $P_{t h, m}$ is estimated from the SWIA ion density $n_{i}$ and temperature $T_{i}$ measurements, and assuming $T_{e} \ll T_{i}$. $P_{t h, m}$ is obtained using

$$
P_{t h, m}=n_{i} k_{B} T_{i}
$$

The total magnetic pressure $P_{B}$ is obtained from the MAG measurements using

$$
P_{B}=B^{2} / 2 \mu_{0}
$$

where $\mu_{0}$ is the vacuum permeability. The crustal field magnetic pressure $P_{B, c}$ is also obtained from equation (3) using $B=B_{c}$, where $B_{c}$ is the magnetic field strength derived from the Morschhauser model (Morschhauser et al., 2014). The solar wind dynamic pressure $P_{d, s w}$ is estimated from both MAVEN/SWIA and MEX/IMA ion density $n_{i}$ and velocity $v_{i}$ using

$$
P_{d}=m_{p} n_{i} v_{i}^{2}
$$

where $m_{p}$ is the proton mass.

\subsection{Case Studies}

Three typical orbits of the 18 orbit segments are shown as examples in Figures 1-9. The figures present the estimated pressure terms, the orbit trajectories, and the particle and magnetic field measurements used to derive the altitudes of the PEB, ICB, and IMB. These three orbits were chosen since they all present different interesting characteristics of the dayside magnetospheres, such as double boundary crossings, crustal field effects, and variations in the relative boundary distances, as described in more detail below. Please note that the solar wind dynamic pressure was close to average, and occasionally low, for all 18 orbit segments. Consequently, the obtained magnetospheric structure represents the system during typical and quiet solar wind conditions.

The orbit trajectories, of the first presented time interval, of both MAVEN (red) and MEX(blue) in Mars Solar Orbital (MSO) coordinates are shown in Figure 1. The MSO system is defined with the $x$ axis toward the Sun, the $y$ axis opposite to the planet velocity vector, and the $z$ axis completing the right-handed system. Figure 1 shows that for this time interval MAVEN moves from the solar wind at SZA $=60^{\circ}$ north of the planet, crossing the magnetosheath and the MPR at SZA $<20^{\circ}$ and entering the ionosphere around SZA $=55^{\circ}$ in the south. The estimated bow shock (solid line) and MPB (dashed line) given by Vignes et al. (2000) are included as a reference.

Figure 2 shows the estimated pressure terms, the $P_{t h, i}$ (orange), $P_{B, c}$ (blue), $P_{B}$ (black), $P_{t h, m}$ (purple), and the $P_{d, s w}$ (gray), as a function of height $h$ above the surface. Panel (b) shows an enlargement of the altitude region $180 \mathrm{~km}\left(1.05 R_{M}\right)$ to $1,200 \mathrm{~km}\left(1.35 R_{M}\right)$ to better show the details of the induced magnetospheric structure. The LPW electron density and temperature, and the SWIA ion density and temperature are used to estimate $P_{t h, i}$ and $P_{t h, m}$. The LPW $n_{e}$ and $T_{e}$ measurement cadences are 4 and $8 \mathrm{~s}$, respectively. The SWIA $n_{i}$ and $T_{i}$ measurement cadence is $4 \mathrm{~s}$. 

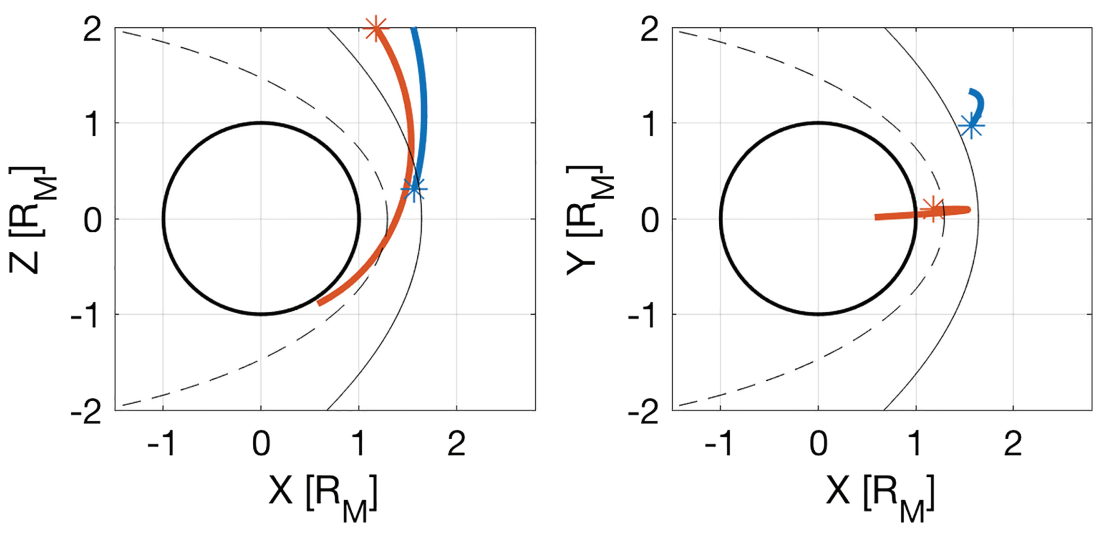

Figure 1. The orbit trajectories for MAVEN (red) and MEX (blue) in MSO coordinates for the time interval 20 September 2015 23:09 to 21 September 2015 00:11. The markers show the beginning of each orbit segments. The black lines mark the bow shock (solid) and magnetic pile-up boundary (dashed) given by Vignes et al. (2000).

Figure 2 shows that the different pressure terms clearly separate four distinct regions. (1) The innermost region is the ionosphere, below around $245 \mathrm{~km}$, where the $P_{t h}$ is the dominant pressure term. (2) Above the ionosphere, between altitudes around 245 to $949 \mathrm{~km}$, the $P_{B}$ is the dominant term since the IMF is piled-up and creates the MPR. (3) Further out the magnetosheath shows a dominant $P_{t h}$ term and (4) outside of the bow shock the dominant pressure term is the $P_{d}$. These structures are present in all the studied orbits, but the altitudes of the various transition regions can change with several hundreds of $\mathrm{km}$.
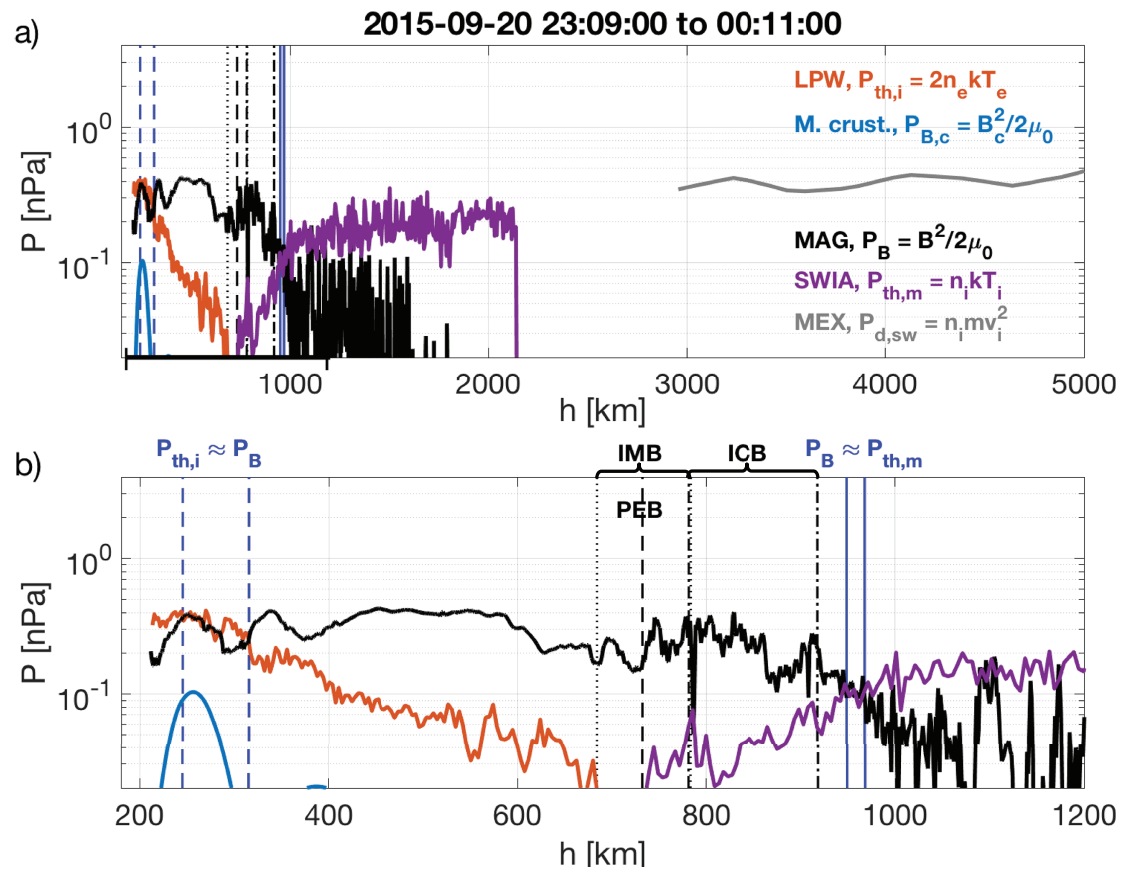

Figure 2. Estimated $P_{t h, i}$ (orange (total $P_{t h, i}$, assuming $T_{i}=T_{e}$ )), $P_{B}$ (black), $P_{t h, m}$ (purple), and $P_{d, s w}$ (gray) against altitude for the date 20 September 2015. The magnetic pressure of the crustal field $P_{B, c}$ (blue) is obtained from the model by Morschhauser et al. (2014). The vertical lines show the altitudes of the boundaries: the inner pressure balance boundary $P_{t h, i} \approx P_{B}$ (blue dashed line) at $245-315 \mathrm{~km}$, the PEB (gray dashed) at $732 \mathrm{~km}$, ICB (gray dash dotted) at 781-918 km, the IMB (gray dotted) at 686-783 km, and the outer pressure balance boundary $P_{B} \approx P_{t h, m}$ (blue solid) at $949-968 \mathrm{~km}$. The altitudes of the pressure balance boundaries (blue vertical lines) are obtained from the data presented in this figure; the altitudes of the PEB, IMB, and ICB (gray vertical lines) are obtained from the data presented in Figure 3. Panel (b) shows an enlargement of the altitude range from 180 to 1,200 km, marked with the black horizontal line on the $x$ axis in panel (a). ICB = ion composition boundary; IMB = induced magnetosphere boundary; LPW $=$ Langmuir Probe and Waves; $\mathrm{MAG}=$ magnetometer investigation; $\mathrm{MEX}=$ Mars Express; $\mathrm{PEB}=$ photoelectron boundary; SWIA = Solar Wind Ion Analyzer. 
Table 1

Summary of the Conditions Used to Obtain the IMB Altitude Estimates from the SWEA Electron Energy Flux Measurements, for the Three Stated Energy Ranges

\begin{tabular}{lccc}
\hline \multirow{2}{*}{ Energy range $[\mathrm{eV}]$} & \multicolumn{3}{c}{ Conditions on the energy flux } \\
\cline { 2 - 4 } & Flux decrease & Minimum $\left[\mathrm{keV} /\left(\mathrm{s} \cdot \mathrm{cm}^{-2} \cdot \mathrm{sr} \cdot \mathrm{keV}\right)\right]$ & Maximum $\left[\mathrm{keV} /\left(\mathrm{s} \cdot \mathrm{cm}^{-2} \cdot \mathrm{sr} \cdot \mathrm{keV}\right)\right]$ \\
\hline $25-37$ & 1.8 & $1.78 \mathrm{E} 9$ & $1.87 \mathrm{E} 9$ \\
$37-66$ & 5 & $3.90 \mathrm{E} 8$ & $2.10 \mathrm{E} 9$ \\
$59-83$ & 10 & $1.10 \mathrm{E} 8$ & $1.30 \mathrm{E} 9$ \\
\hline
\end{tabular}

Note. IMB = induced magnetosphere boundary; SWEA = Solar Wind Electron Analyzer.

Figure 2 shows that for this particular trajectory $P_{B, c}<P_{B}$; that is, MAVEN is in a region with weak crustal fields that has a small impact on the $P_{B}$. The crustal field strength impacts the lower altitude, at around $245 \mathrm{~km}$, of the pressure balance boundary, where $P_{t h, i} \approx P_{B}$, but do not effect the upper altitude, at around $315 \mathrm{~km}$, substantially. The $P_{t h, i} \approx P_{B}$ boundary is marked with the two blue vertical dashed lines. We use a simple definition of the pressure balance boundary altitude, the boundary ranges from the lowest altitude where $P_{t h, i}=P_{B}$ until the highest altitude. How the two pressure terms are varying within this altitude range is not taken into consideration. For the 18 studied orbit segments the maximum difference found was $<0.2 \mathrm{nPa}$. The altitudes and SZAs of the lower pressure balance boundaries for all 18 orbit segments are presented in Table 1 Column 2. Above the pressure balance boundary is the MPR that is a region of increased magnetic field strength and low magnetic field fluctuations compared to the magnetosheath. In the altitude region between 700 and $900 \mathrm{~km}$, where the estimated thermal pressure is increasing due to the shocked solar wind particles in the magnetosheath, is where we detect the other commonly used plasma boundaries: the IMB, PEB, and ICB. The altitudes of the IMB, PEB, and ICB (gray vertical lines) are estimated from the data presented in Figure 3. A detailed description of the definitions used to obtain these boundary altitudes is presented in section 3.1.1.

The uppermost boundary presented in Figure 2 is the pressure balance boundary where $P_{B} \approx P_{t h, m}$. The boundary is found at 949 to $968 \mathrm{~km}$ for this orbit segment. This is the uppermost boundary for all the 18 orbit segments. The altitudes and SZAs of the $P_{B} \approx P_{t h, m}$ boundary of all 18 orbit segments are presented in Table 1 Column 6. Figure 2a also shows the solar wind dynamic pressure obtained from the MEX/IMA measurements (gray line) for altitudes above $3,000 \mathrm{~km}$. The MEX/IMA measurement cadence is $60 \mathrm{~s}$.

\subsubsection{The PEB, ICB, and IMB}

Previous missions and studies have identified and characterized different boundaries present on the dayside of the Martian induced magnetosphere: the PEB, the ICB, and the IMB. In this section we identify these boundaries and compare their altitudes. The altitudes of the PEB, ICB, and IMB are estimated with the methods described below.

The position of the PEB is obtained from the SWEA energy spectrograms. The PEB altitude has been estimated by eye due to the problems encountered when developing an automatic detection routine; see Garnier et al. (2017). The PEB is estimated as the altitude where the photoelectron energy peaks appear (located between 21 and 24 and at $27 \mathrm{eV}$ ) and a large drop of energetic electron fluxes found in the magnetosheath are measured. The photoelectron energy peaks are due to ionization of $\mathrm{CO}_{2}$ by 30.4-nm radiation (Mantas \& Hanson, 1979). See Garnier et al. (2017) for a detailed description of the detection of PEBs. The PEB is usually a narrow boundary region where the electron energy spectrum changes from ionospheric-like to magnetosheath-like within a km, which is why the PEB altitudes listed in Table 2 Column 3 are usually given for a single altitude. Occasionally, the electron spectra have the appearance of a transition region, meaning that it is difficult to differentiate between ionospheric-like and magnetosheath-like. For these time intervals an altitude range has been stated.

The altitude of the PEB in Figures 2 and 3 is given by the dashed gray line at $732 \mathrm{~km}$ and is estimated from the SWEA energy-time spectrogram presented in Figure 3b. The SWEA spectrogram shows that the electron population changes from solar wind-like to magnetosheath-like at around 23:46:30, which is the bow shock crossing. A second clear transition is occurring at around 00:00:30 where the photoelectron energy peaks are first detected and the energetic electron fluxes drop, which is the characteristics of the PEB. The SWEA 


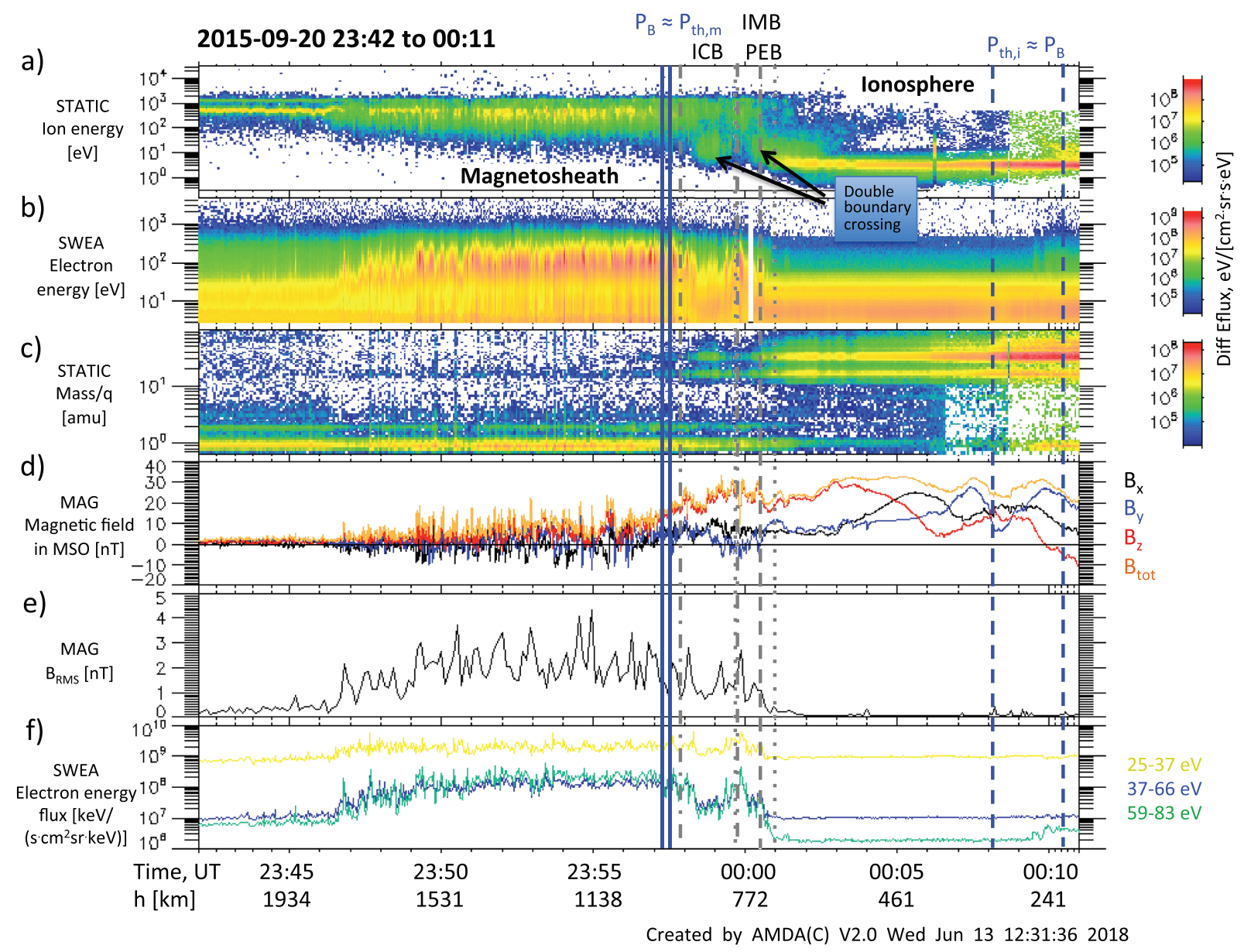

Figure 3. From top to bottom: (a) MAVEN/STATIC energy-time spectrogram of omnidirectional ion energy flux (C0 mode), (b) MAVEN/SWEA energy-time spectrogram of omnidirectional electron energy flux, (c) MAVEN/STATIC mass-time spectrogram of omnidirectional energy flux (C6 mode), (d) MAVEN/MAG magnetic field $B_{x}$ (black), $B_{y}$ (blue), $B_{z}$ (red), and $B_{\text {tot }}$ (orange), (e) RMS of the magnetic field magnitude, (f) SWEA electron energy fluxes for the energy ranges 25-37 eV (yellow), 37-66 eV (blue), and 59-83 eV (green). The vertical lines give the times of the boundary crossings: the outer pressure balance boundary $P_{B} \approx P_{t h, m}$ (solid blue), ICB (dash-dotted gray), the IMB (dotted gray), the PEB (dashed gray), and the inner pressure balance boundary $P_{t h, i} \approx P_{B}$ (dashed blue). Please note that the lower altitude of the ICB almost coincides with the upper altitude of the IMB at around 23:59:40. ICB = ion composition boundary; $\mathrm{IMB}=$ induced magnetosphere boundary; MAG = magnetometer investigation; MAVEN = Mars Atmosphere and Volatile Evolution; PEB = photoelectron boundary; STATIC = Suprathermal and Thermal Ion Composition; SWEA = Solar Wind Electron Analyzer.

measurements are given with 2-s time steps. Each spectrum is studied separately to obtain the PEB altitude, as described in detail in Garnier et al. (2017).

The position of the ICB is obtained, similar to the convention used by Matsunaga et al. (2017), from searching for the ion number density ratio $r_{i}=1$, where

$$
r_{i}=\left(n_{\mathrm{O}^{+}}+n_{\mathrm{O}_{2}^{+}}\right) /\left(n_{\mathrm{H}^{+}}+n_{\mathrm{He}^{++}}\right)
$$

The ICB is located above $400 \mathrm{~km}$ for all of the studied time intervals. Above $400 \mathrm{~km}\left(n_{\mathrm{O}^{+}}+n_{\mathrm{O}_{2}^{+}}\right)>10 n_{\mathrm{OH}^{+}}$, with $\mathrm{OH}^{+}$being the third most common ion species on the dayside around the ICB altitudes (Benna et al., 2015). The $\mathrm{OH}^{+}$density will therefore have a negligible effect on the ICB altitude estimation and is not included for simplicity.

The altitude of the ICB in Figures 2 and 3 is given by the dash-dotted gray lines at 781 and $918 \mathrm{~km}$ and is estimated from the STATIC ion density measurements and ion mass spectrogram presented in Figure 3c. The STATIC basic measurement cadence is $4 \mathrm{~s}$.

One especially interesting feature can be seen in the spectrogram, marked with the two arrows in Figure 3a, between 23:58:00 and 23:59:30. The feature is seen as a drop in energetic ion energy fluxes and an increase 
in the flux of ions of 10 to $100 \mathrm{eV}$ (Figure 3a), which is related to an increase in ionospheric ions of higher masses and a decrease in solar wind ions (Figure 3c), and a decrease in energetic electron energy fluxes (Figures $3 \mathrm{~b}$ and $3 \mathrm{f}$ ). The feature shows a double boundary crossing. As the spacecraft moves toward the planet it encounters a region that looks more ionospheric-like. This could be due to spatial or/and temporal changes. For example, the transition region from the magnetosheath to the ionosphere is not symmetrical and the spacecraft enters into a region where the ionosphere can be detected at higher altitude or the boundary structure is very dynamic and the altitude is changing during the time interval when the spacecraft is passing this region, or a combination of the two. It is also possible that the feature is the signature of a plasma cloud related to plasma loss from Mars' ionosphere, as discussed in Halekas et al. (2016); however, the event does not have the typical plasma cloud appearance. The only way to conclude if the double boundary crossing is a temporal or a spatial variation, or both, is by combining measurements from multiple spacecraft that crosses the region at various positions in time and space. Unfortunately, the limited number of current Mars orbiters provide very few, if any, possibilities for such studies. Nevertheless, when analyzing hundreds of MAVEN orbits, we encountered double boundary crossings regularly and can therefore conclude that double boundary crossings are very common in the measurements from the Martian dayside induced magnetosphere. It is therefore important to acknowledge their presence and to also present double boundary crossings in a study of the structure of the Martian induced magnetosphere.

The last boundary presented in this study is the IMB. The altitude of the IMB is obtained from the MAG and SWEA data. The measurement frequency of MAG is $32 \mathrm{~Hz}$, but for the plotting we have used the $1 \mathrm{~Hz}$ averaged data. We use SWEA data from three different energy ranges: 25 to 37, 37 to 66, and 59 to $83 \mathrm{eV}$. Inspection showed this range to be where the largest decrease in electron energy flux was detected, which is in agreement with previous findings (e.g., Bertucci et al., 2003). The decrease in electron fluxes in these energy ranges has previously been studied by Crider et al. (2000). They used a model of the effect of the electron impact ionization (EII) on the electron energy distribution to show that EII can reproduce the detected decrease in the electron fluxes. The energy ranges corresponds to the peak values of the cross sections for EII of neutral hydrogen and oxygen, the dominant neutral species at these altitudes, which can explain why the decrease in electron energy flux is largest for the above energy ranges.

The IMB is the most complex of all boundaries included in this study, partly because several parameters were included and partly because earlier descriptions of the boundary could not be confirmed. From the MGS measurements the MPB/IMB was described as the beginning of the MPR where the magnetic field strength can suddenly increases with a factor 2 to 3 and the electron fluxes dramatically drops (Nagy et al., 2004). However, it was noted that the increase in field magnitude was occasionally hard to detect near LT noon, which is where most of our case studies are located. This was confirmed by our 18 case studies that commonly show slowly increasing magnetic field strengths, over a distance of 200-550 km, starting hundreds of $\mathrm{km}$ before the electron energy flux drop. Only two orbits showed the increase in $B$ at the same altitude as the drop in electron energy fluxes. This seems to be the general case since later studies tend to use the fluctuation of the field instead of the increase as a condition for the MPB/IMB (e.g., Matsunaga et al., 2017). That is also a good argument for referring to this boundary as the IMB instead of MPB since the drop in electron energy fluxes are more often found within the MPR. For a measure of the magnetic field fluctuation we use the root-mean-square (RMS) value of the magnitude of the magnetic field, which was also done by Acuña et al. (1998) when detecting the bow shock and MPB from MGS magnetic field measurements. The MAVEN/MAG magnetic field RMS is estimated for each $8 \mathrm{~s}$. Fifteen of the 18 orbits showed a decrease in the RMS of the magnetic field of at least a factor 5 during the same altitude as the drop in the electron energy flux. Three orbits showed no, or a smaller, decrease at the altitudes of the drop in electron energy flux; instead, they showed a more prominent decrease at lower altitudes.

The conditions used to detect the IMB are listed in Table 1. The first column gives the chosen energy ranges for the SWEA electron energy flux measurements, 25 to 37, 37 to 66, and 59 to $83 \mathrm{eV}$. The second column states the required drop in the energy flux, given as a factor. The third column gives the minimum energy flux value before the drop, and the fourth column states the maximum value of the energy flux after the drop, which need to be maintained for at least $200 \mathrm{~km}$ below the end of the drop in order to avoid double boundary crossings. Only data recorded below $1,400 \mathrm{~km}$ are used. If a minimum of six of the conditions stated in Table 1 are fulfilled and the RMS of the magnetic field magnitude is decreasing with at least a factor of 1.6 over the detected altitude range, then that altitude range is given as the IMB. The derived values are based on inspecting the boundaries and deriving a range that detect a boundary that is in reasonable 

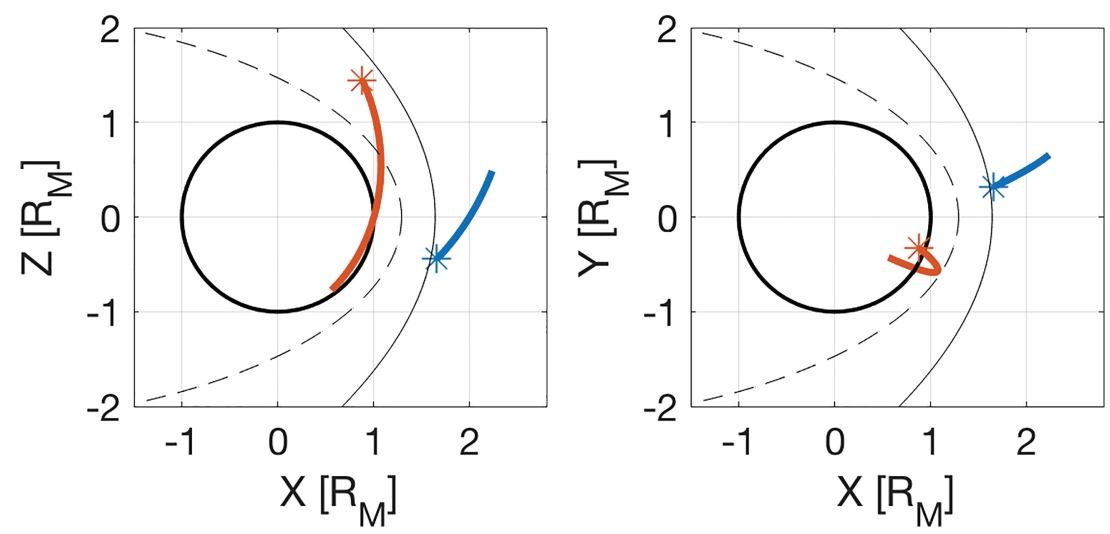

Figure 4. The orbit trajectories for MAVEN (red) and MEX (blue) in MSO coordinates for the time interval 23 October 2015 00:20 to 00:58. The plot setup is the same as in Figure 1.

agreement with what would be estimated by eye. It is therefore likely that they would not be sufficient for a data set larger than the 18 studied orbit segments listed in Table 2, but it is reasonable to assume that they still give a good guideline for boundary detection close to the subsolar point.

The altitude of the IMB in Figures 2 and 3 is given by the dotted gray lines at 686 and $783 \mathrm{~km}$ and is estimated from the SWEA electron energy flux measurements and MAG magnetic field measurements presented in Figures $3 \mathrm{~d}-3 \mathrm{f}$. In Figure 2 it is clearly shown that the fluctuations of the magnetic field are decreasing within the defined IMB region. Figure 2 also shows that the increase in the magnetic field strength that marks the beginning of the MPR is located between 900 and 1,000 km, which is about $100 \mathrm{~km}$ above the altitude where we detect the beginning of the electron energy flux drop and the decrease in magnetic field fluctuations. The decreases in the electron energy flux of the three chosen energy ranges are shown in Figure 3f. The decrease in the RMS of the magnetic field magnitude is shown in Figure 3e.

The bow shock altitude could also be used for a comparison, but for all the case studies listed in Table 2 only four include the crossing of the bow shock.

The orbit trajectories of the second presented time interval, 23 October 2015 00:20 to 00:58, of both MAVEN (red) and MEX (blue) are shown in Figure 4. For this time interval MAVEN moves from the magnetosheath at $\mathrm{SZA}=60^{\circ}$ in the northern hemisphere, crossing the magnetosheath and the MPR with a minimum $\mathrm{SZA}=30^{\circ}$ at $623 \mathrm{~km}$ and entering the ionosphere around SZA $=45^{\circ}$ in the southern hemisphere. The estimated bow shock (solid) and MPB (dashed) given by Vignes et al. (2000) are included as a reference.

The estimated pressure terms, for the time interval 23 October 2015 00:20 to 00:58, are presented in Figure 5. Figure 5a once again shows the clear distinction between the different regions dominated by the $P_{t h, i}, P_{B}, P_{t h, m}$, and $P_{d, s w}$. At around $200 \mathrm{~km}$ the piled-up magnetic field decreases in a manner similar to the magnetospheric structure of Venus, which lacks a global magnetic field and does not posses any known crustal magnetic fields. Figure $5 \mathrm{~b}$ shows that the spacecraft encounters a stronger crustal field region at altitude $170 \mathrm{~km}$. The inner pressure balance boundary $P_{t h, i} \approx P_{B}$ (blue dashed line) is found at 189-190 km, an altitude which is not strongly affected by any strong crustal fields nearby. The outer pressure balance boundary $P_{B} \approx P_{t h, m}$ (blue solid) are detected at $624-647 \mathrm{~km}$. In between the pressure boundaries are the IMB, PEB, and ICB, which are detected using the data presented in Figure 6 and also included in Figure 5 for comparison.

The altitude of the PEB in Figures 5 and 6 is given by the dashed gray line at $409-434 \mathrm{~km}$ as determined from the SWEA energy-time spectrogram presented in Figure $6 \mathrm{~b}$. The SWEA spectrogram shows that the electron population is typically magnetosheath-like before 00:44:40 and then changes to a transition region, which is neither magnetosheath-like nor ionosphere-like before the photoelectrons can be detected between 00:47:25 and 00:47:50, and the appearance turns into ionosphere-like. Each spectrum has been studied separately to obtain the correct boundary altitudes. The altitude of the ICB in Figures 5 and 6 is given by the dash-dotted gray lines at 551 and $555 \mathrm{~km}$. The ICB altitude is estimated from the STATIC ion density measurements and ion mass spectrogram presented in Figure 6c. The altitude of the IMB presented in Figures 5 and 6 is given by the dotted gray lines at 346 and $575 \mathrm{~km}$ and is estimated from the MAG and SWEA data, using the method 
a)

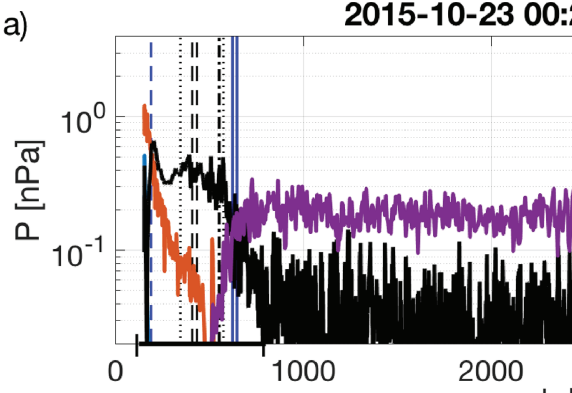

2015-10-23 00:20:00 to 00:58:00

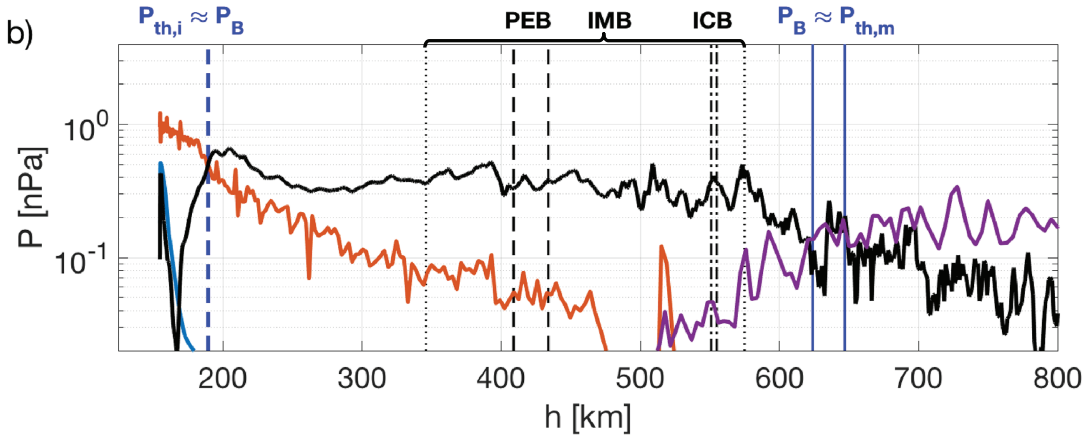

Figure 5. Pressure terms and boundaries estimated for 23 October 2015. The plot setup is the same as in Figure 2. The boundary locations are estimated to the following altitudes: the inner pressure balance boundary $P_{t h, i} \approx P_{B}$ (blue dashed line) at 189-190 km, the PEB (gray dashed) at 409-434 km, ICB (gray dash dotted) at 551-555 km, the IMB (gray dotted) at $346-575 \mathrm{~km}$, and the outer pressure balance boundary $P_{B} \approx P_{t h, m}$ (blue solid) at 624-647 km. The altitudes of the pressure balance boundaries (blue vertical lines) are obtained from the data presented in this figure; the altitudes of the PEB, IMB, and ICB (gray vertical lines) are obtained from the data presented in Figure 6. Panel (b) shows an enlargement of the altitude range from 154 to $800 \mathrm{~km}$, marked with the black horizontal line on the $x$ axis in panel (a). Please note that for this orbit both the PEB and ICB are found within the IMB. LPW = Langmuir Probe and Waves; $\mathrm{MAG}=$ magnetometer investigation; SWIA $=$ Solar Wind Ion Analyzer; MEX = Mars Express; $\mathrm{PEB}=$ photoelectron boundary; IMB = induced magnetosphere boundary; ICB = ion composition boundary.

described above. For this orbit the IMB is shown to be spanning over more than $200 \mathrm{~km}$. However, there are several potential causes of the large boundary thickness. For example, the spacecraft could be grazing within the boundary for an extended time period, the boundary could be moving in the same direction as the spacecraft, and/or the boundary is not symmetrical around the planet and the spacecraft could be moving from a region where the boundary is located at higher altitudes to a region where it is located at lower altitudes. These complications apply to all boundary detections and the width of the boundary should therefore be used with care.

The third presented time interval is from 28 November 2016 from 23:05 to 23:49. The orbit trajectories are presented in Figure 7, and the measurements are shown in Figures 8 and 9. The spacecraft enters a very strong crustal field region at $315 \mathrm{~km}$, where the magnetic pressure starts to increase rapidly. This orbit shows a similar magnetospheric structure as the previously presented, but the IMB is found at a higher altitude, approaching the altitude of the upper pressure balance boundary at $972 \mathrm{~km}$. The presence of the IMB at higher altitudes than the PEB and ICB is a common feature, as can be seen from studying the boundary altitudes listed in Table 2.

Another common feature is the order of the estimated boundaries; in this time interval the boundaries occur at separate altitudes and in a certain order. From low altitudes to high we find the boundaries in the order $P_{t h, i} \approx P_{B}$, PEB, ICB, IMB, and then $P_{B} \approx P_{t h, m}$, which is also the most common magnetospheric structure in the 18 studied orbit segments; see Table 2. Table 2 lists the time intervals of the 18 case studies, if the orbit segment is from the inbound or outbound part of the orbit, and the estimated altitudes and SZAs of the boundaries. The mean and median values, listed in Table 3, of the boundary altitudes confirm that the boundaries have the same "preferred" order as presented in Figure 8, with an occasional overlap of the lower and upper limits of two neighboring boundaries. Figures 3, 6, and 9 also confirm the simulations results by 


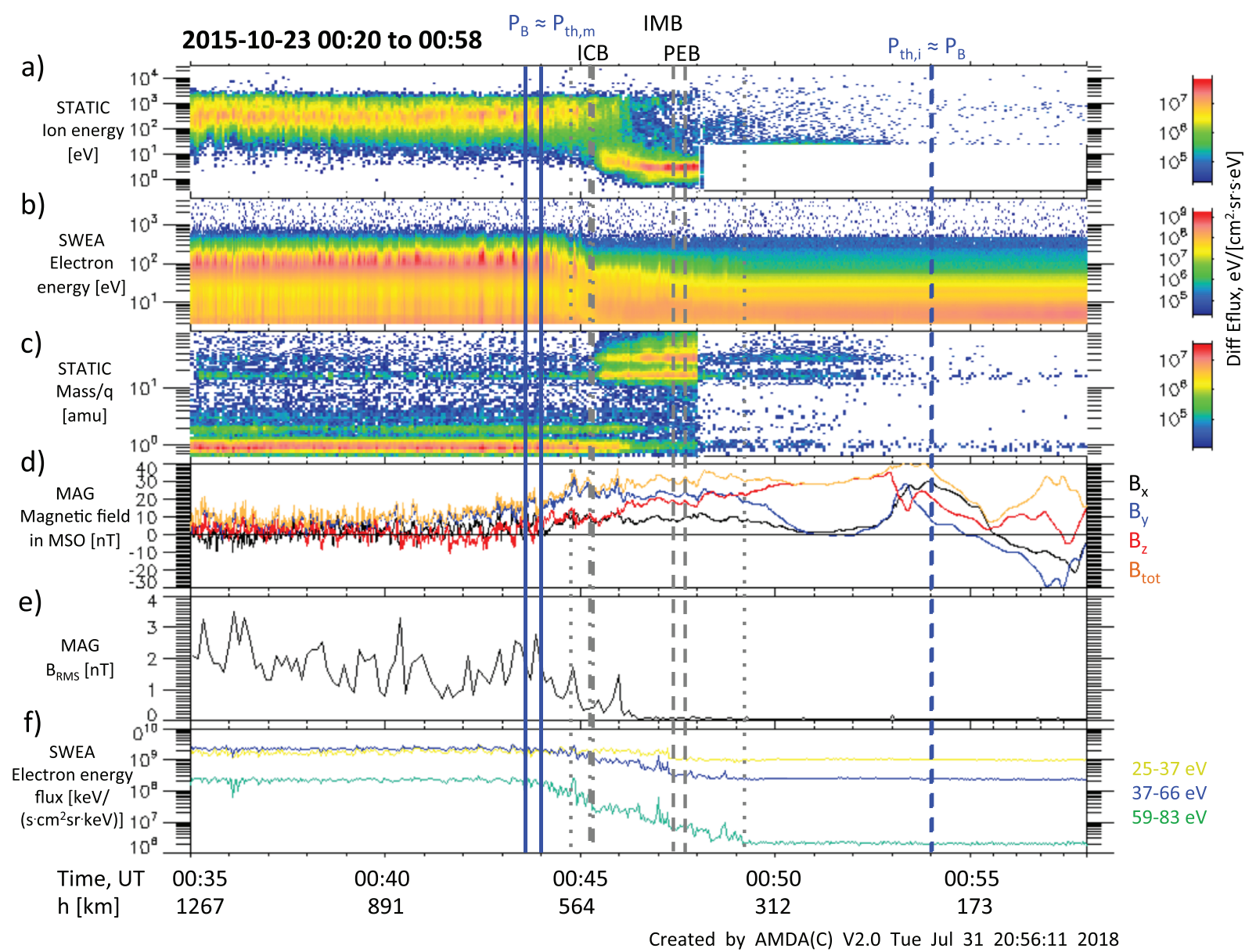

Figure 6. The plot setup is the same as in Figure 3.

Bößwetter et al. (2004) that the ICB is located within the MPR and not at the same location as the MPB. Bößwetter et al. (2004) used a 3-D hybrid model to study the bow shock, ICB, and MPB at Mars.

The boundary altitudes listed in Table 3 should be considered as simple indicators of the common altitudes of the boundaries. The boundary altitudes listed in Table 2 shows that each boundary can vary with hundreds of $\mathrm{km}$ from orbit to orbit. In addition, due to the small amount of available orbits when MEX can be used as
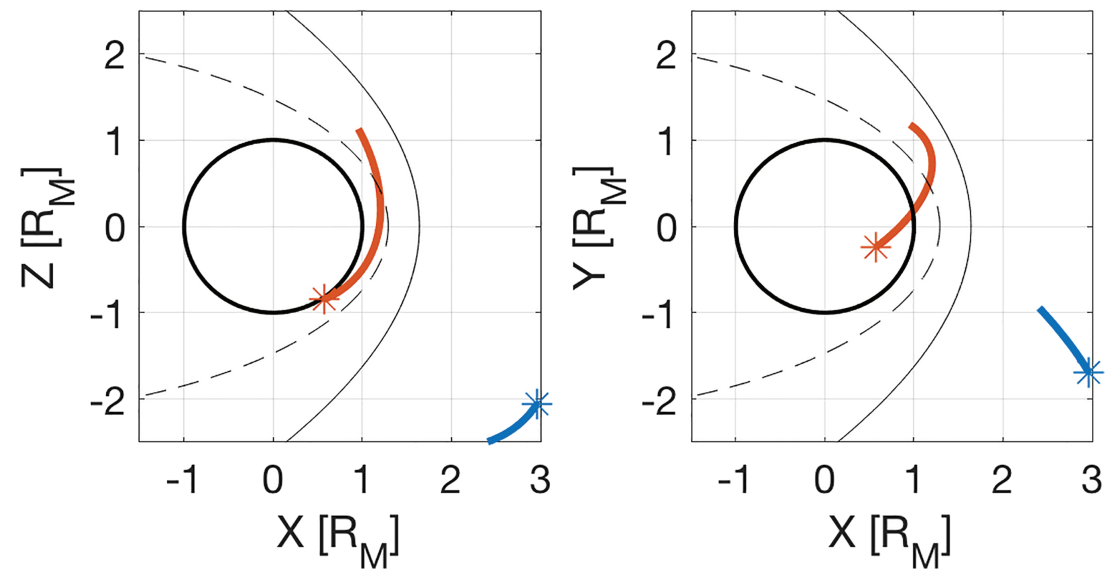

Figure 7. The orbit trajectories for MAVEN (red) and MEX (blue) in MSO coordinates for the time interval 28 November 2016 23:05 to 23:49. The plot setup is the same as in Figure 1. 
Table 2

Boundary Altitudes for the 18 Orbit Segments When Both MAVEN and MEX were Within $60^{\circ} \mathrm{SZA}$

\begin{tabular}{|c|c|c|c|c|c|}
\hline \multirow[b]{3}{*}{ Date } & \multicolumn{5}{|c|}{ Boundary altitudes $[\mathrm{km}]$} \\
\hline & \multicolumn{5}{|c|}{ SZA [deg] } \\
\hline & $P_{t h, i} \approx P_{B}$ & PEB & ICB & IMB & $P_{B} \approx P_{t h, m}$ \\
\hline $2014-11-17^{a}$ & $338-343$ & $404-665$ & $623-751$ & $648-787$ & $1347-1621$ \\
\hline 15:06-15:55 & $56-55$ & $51-38$ & $40-35$ & $39-34$ & $23-22$ \\
\hline 2015-03-16 & $238-264$ & 615 & $633-637$ & $547-675$ & $706-940$ \\
\hline 19:04-19:45 & $47-44$ & 31 & 31 & $32-31$ & $31-30$ \\
\hline 2015-03-17 & $349-366$ & 409 & $474-706$ & $643-815$ & $849-857$ \\
\hline 08:52-09:18 & $38-37$ & 35 & $33-30$ & 30 & 30 \\
\hline 2015-03-17 & $304-449$ & 711 & $747-751$ & $721-1,014$ & $1,000-1019$ \\
\hline $17: 39-18: 20$ & $40-33$ & 30 & 30 & $30-32$ & 32 \\
\hline 2015-03-20 & $263-264$ & 515 & $539-565$ & $496-731$ & $741-1,325$ \\
\hline 08:55-09:35 & 38 & 28 & $28-27$ & 28 & $28-37$ \\
\hline $2015-03-23$ & $229-326$ & 697 & $761-766$ & $693-895$ & $1,739-2,149$ \\
\hline $13: 43-14: 21$ & $36-29$ & 26 & 27 & $25-29$ & $48-56$ \\
\hline $2015-09-20$ & $245-315$ & 732 & $781-918$ & $686-783$ & 949-968 \\
\hline 23:09-00:11 & $53-46$ & 21 & $19-13$ & 23-18 & $12-11$ \\
\hline $2015-09-22$ & $208-248$ & 761 & $796-801$ & $721-798$ & $793-799$ \\
\hline $02: 57-03: 32$ & $56-51$ & 18 & 17 & $20-17$ & 17 \\
\hline $2015-09-28$ & $323-324$ & 837 & $839-844$ & $840-995$ & $965-990$ \\
\hline $13: 25-14: 17$ & 38 & 10 & 10 & $10-6$ & 6 \\
\hline 2015-09-29 & $297-415$ & 1026 & $1033-1038$ & $1027-1171$ & $1155-1169$ \\
\hline 03:05-04:01 & $40-31$ & 6 & 6 & 6 & 6 \\
\hline $2015-10-23$ & $189-190$ & $409-434$ & $551-555$ & $346-575$ & $624-647$ \\
\hline 00:20-00:58 & 45 & 32 & 30 & $34-30$ & 30 \\
\hline $2016-11-19^{a}$ & $218-268$ & $373-634$ & $581-638$ & $646-739$ & $703-766$ \\
\hline $12: 32-13: 07$ & $40-37$ & 33 & $32-33$ & $33-34$ & $34-35$ \\
\hline $2016-11-20^{\mathrm{a}}$ & $186-187$ & 450 & $457-461$ & 467-557 & $671-741$ \\
\hline 02:25-03:00 & 45 & 32 & 32 & 32 & $33-34$ \\
\hline $2016-11-25^{\mathrm{a}}$ & 321 & - & $418-485$ & $1008-1295$ & $1276-1617$ \\
\hline $11: 52-12: 33$ & 34 & - & $30-28$ & $30-35$ & $35-41$ \\
\hline $2016-11-28^{a}$ & $368-373$ & $491-630$ & $665-670$ & $698-831$ & $972-1048$ \\
\hline $23: 05-23: 49$ & $33-32$ & $27-24$ & 23 & $23-24$ & $24-25$ \\
\hline $2016-11-29^{a}$ & $288-289$ & $433-502$ & $455-468$ & $496-712$ & $714-991$ \\
\hline $17: 34-18: 19$ & 38 & $29-26$ & $28-27$ & $26-22$ & $22-24$ \\
\hline $2017-06-25^{\mathrm{a}}$ & $269-270$ & 607 & $563-644$ & $607-665$ & $623-657$ \\
\hline $12: 53-13: 29$ & 49 & 34 & $36-34$ & $34-33$ & $34-33$ \\
\hline $2017-06-28^{a}$ & 255 & 511 & $503-510$ & $497-601$ & $555-591$ \\
\hline $17: 54-18: 33$ & 53 & 41 & 41 & $41-39$ & $40-39$ \\
\hline
\end{tabular}

Note. MAVEN = Mars Atmosphere and Volatile Evolution; MEX = Mars Express; SZA = solar zenith angle; $\mathrm{PEB}=$ photoelectron boundary; $\mathrm{ICB}=$ ion composition boundary; IMB = induced magnetosphere boundary. Dates are formatted as YYYY-MM-DD.

${ }^{\mathrm{a}}$ Inbound orbits. 
a)
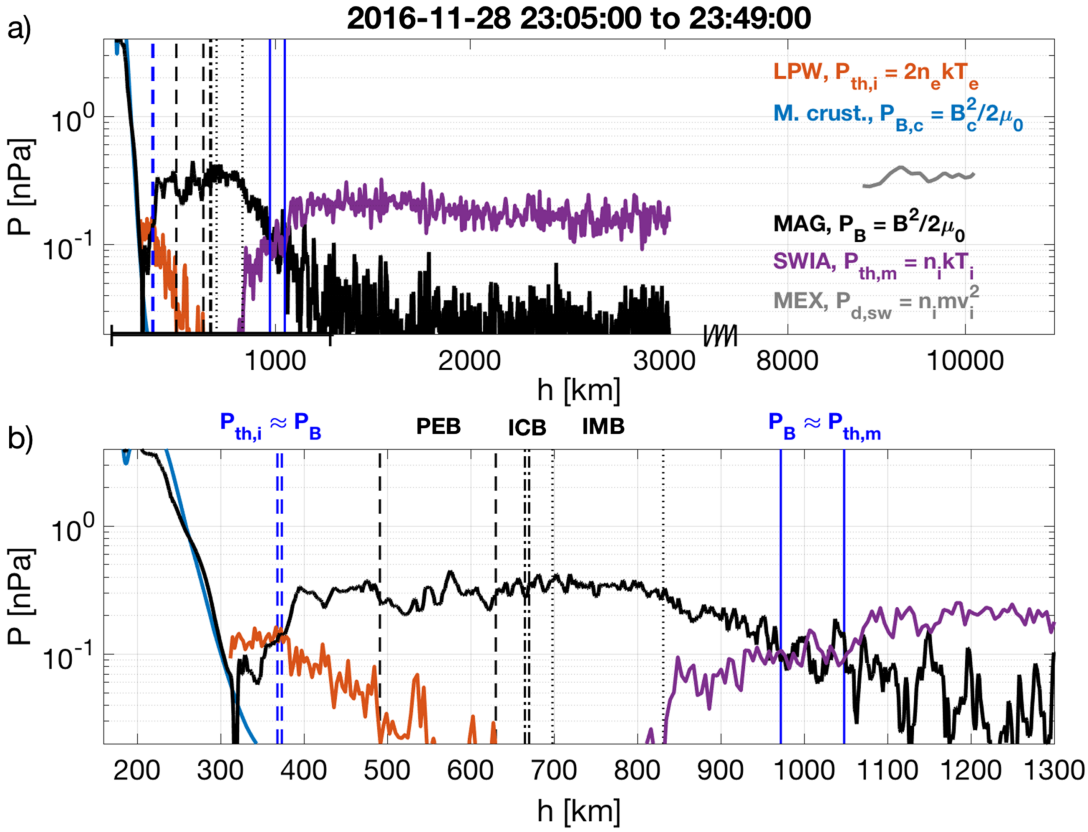

Figure 8. Pressure terms and boundaries estimated for 28 November 2016. The plot setup is the same as in Figure 2. The boundary locations are estimated to the following altitudes: the inner pressure balance boundary $P_{t h, i} \approx P_{B}$ (blue dashed line) at 368-373 km, the PEB (gray dashed) at 491-630 km, ICB (gray dash dotted) at 665-670 km, the IMB (gray dotted) at $698-831 \mathrm{~km}$, and the outer pressure balance boundary $P_{B} \approx P_{t h, m}$ (blue solid) at $972-1,048 \mathrm{~km}$. The altitudes of the pressure balance boundaries (blue vertical lines) are obtained from the data presented in this figure; the altitudes of the PEB, IMB, and ICB (gray vertical lines) are obtained from the data presented in Figure 9. Panel (b) shows an enlargement of the altitude range from 160 to $1,300 \mathrm{~km}$, marked with the black horizontal line on the $x$ axis in panel (a). Please note that the $x$ axis of panel a has been cut between 3,500 and 7,500 km.

a solar wind monitor at the same time as MAVEN is measuring the dayside magnetospheric structure, the boundaries are compared over a larger range of SZA. For the lower boundaries this is of minor importance since they have a weak dependence on SZA, but for the upper boundaries this could be a potential problem. However, other parameters are also important for the boundary positions. For example, excluding low $(<20)$ or high ( $>40)$ SZA for the upper pressure balance boundary only changes the average altitudes with a few tens of $\mathrm{km}$. In fact, the SZA dependence on the boundary altitudes cannot easily be detected in the studied orbits, since its dependence is imbedded together with the influence of other driving factors such as, for example, the solar wind dynamic pressure, EUV flux, LT, crustal fields strength, and the IMF strength and direction. Deducing the influence of each of these parameters requires a larger number of orbits and is beyond the scope of this article. We have therefore included all of the studied orbits, independent of the SZA of the boundary location, in the averages presented in Table 3. Despite this, the estimated boundary altitudes are in agreement with results from earlier studies. For example, the average PEB altitude of 587-632 km and median of 515-630 km agrees well with the average PEB altitude of $573 \mathrm{~km}$, obtained from almost 1,700 PEB crossings, presented by (Garnier et al., 2017).

\subsection{Statistical Study}

The case studies make it possible to see the details of each orbit segment and to find the altitudes and relative distances of the various boundaries. However, despite the relatively large number of studied orbits, it is not confirmed that they are a good representation of the system in general. The orbit segments are only chosen using the condition that MAVEN and MEX are within SZA $60^{\circ}$, that the lower pressure balance boundary is present (i.e., low crustal field regions), and that the best-quality data from each instrument are available. This should not provide any bias in terms of, for example, EUV influx or IMF direction, and the orbit segments are evenly distributed in time; therefore, it is reasonable to assume that they represent the system well. To confirm this, a statistical study was performed to estimate $P_{t h, i}, P_{B}, P_{t h, m}$, and $P_{d, s w}$ for low SZA. The data were limited to MAVEN measurements performed within a cylinder shape close to SZA $0^{\circ}$ during the time interval from 8 October 2014 to 29 April 2018. For the $P_{t h, i}, P_{B}$, and $P_{t h, m}$ estimates we use a cylinder with radius $0.45 R_{M}$ centered at the subsolar line and with varying length. The outer pressure 


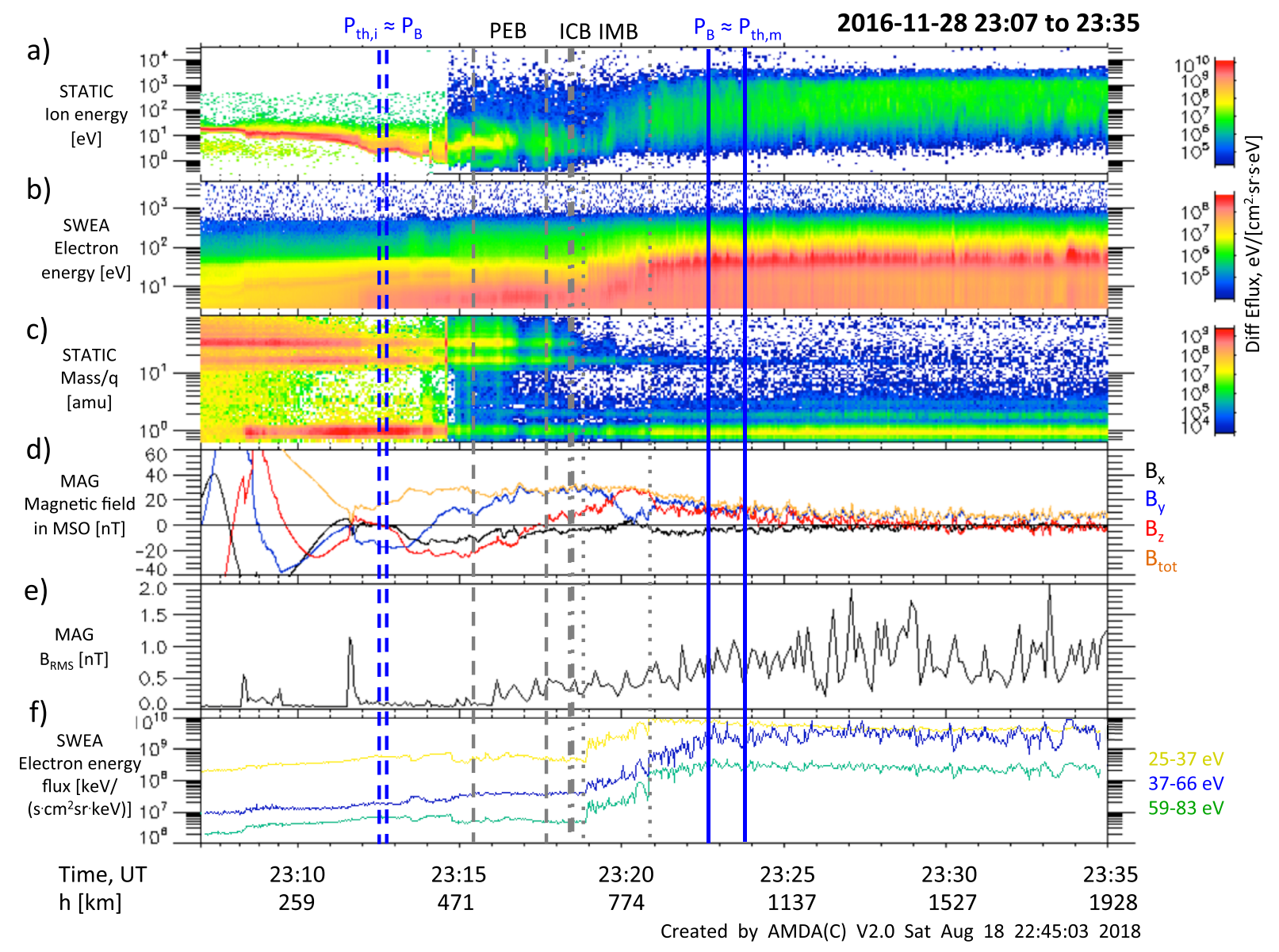

Figure 9. The plot setup is the same as in Figure 3.

term $P_{d, s w}$ is collected from a larger volume represented by a cylinder with radius $0.9 R_{M}$. The two volumes are illustrated in Figure 10, which also shows an example orbit where the red orbit segment represents the section used for the statistical study.

For the $P_{t h, i}$ we use all LPW $n_{e}$ and $T_{e}$ measurements recorded within a cylinder reaching from 120 to $500 \mathrm{~km}$ in altitude above the surface, and with a radius of $0.45 R_{M}$. The results are presented in Figure 11, which shows the mean (red) and the median (yellow) values for each 10-km bin. For the altitudes above $175 \mathrm{~km}$ the pressure shows a steady decrease, but for the pressure below $175 \mathrm{~km}$ the average and median pressure are more uneven. This is due to the small number of orbits covering the lower altitudes. Despite the measurements being recorded during around 2.5 years time, due to the orbit configuration of MAVEN the data set below $175 \mathrm{~km}$ is divided into only two different time intervals. The higher pressures were recorded during 120 orbits from 17 April to 8 May 2015, and the lower pressures were recorded during 172 orbits from 19 October to 19 November 2017. The pressure difference between the two time intervals shows the difference in density due to varying solar EUV flux, caused both by the varying solar activity and the varying distance between Mars and the Sun. The pressure values measured in 2015 were closer in time to the solar maximum in 2014 and 2015 and for closer distances to the Sun, around 1.48 to $1.50 \mathrm{AU}$. The pressure measurements

Table 3

Mean and Median Altitudes for the Boundaries Presented in Table 2

\begin{tabular}{lccccc}
\hline Boundary & $P_{t h, i} \approx P_{B}$ & PEB & ICB & IMB & $P_{B} \approx P_{t h, m}$ \\
\hline Mean altitude $[\mathrm{km}]$ & $272-304$ & $587-632$ & $634-678$ & $654-813$ & $910-1,050$ \\
Median altitude $[\mathrm{km}]$ & $266-302$ & $515-630$ & $602-657$ & $647-785$ & $821-979$ \\
\hline
\end{tabular}

Note. $\mathrm{PEB}=$ photoelectron boundary; ICB $=$ ion composition boundary; IMB = induced magnetosphere boundary. 


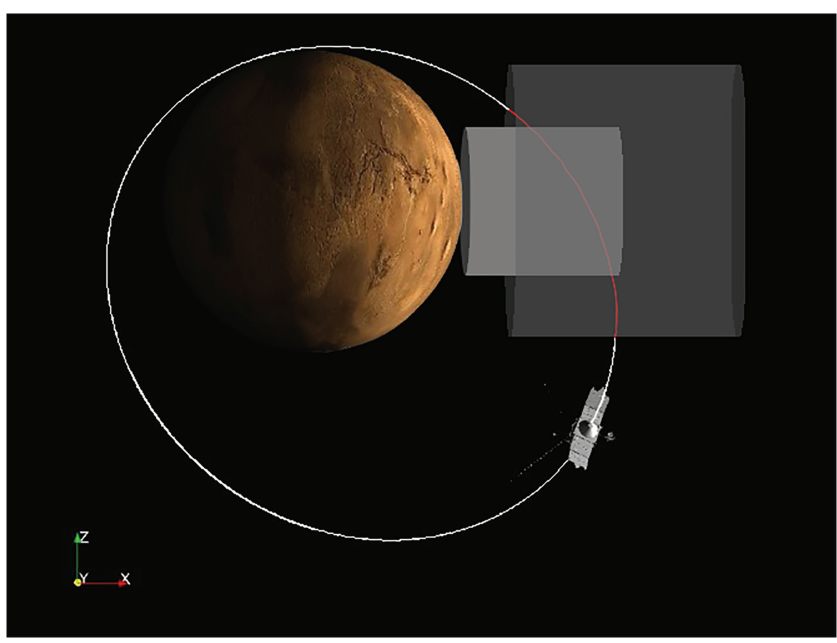

Figure 10. Illustration of the data selection criterion in the MSO coordinate system. The $P_{t h, i}, P_{B}$, and $P_{t h, m}$ presented in Figures 11-13 are estimated from measurements recorded within a cylinder of $0.45 R_{M}$ (light gray cylinder) centered at the subsolar line and the $P_{d, s w}$ measurements are recorded within a cylinder of $0.9 R_{M}$ (dark gray cylinder). The portion of the orbit that are used for the estimates are marked in red. The cylinders are to scale with respect to the planet.

from 2017 were closer to the solar minimum that we are currently approaching and for larger distances to the Sun, around 1.66 to $1.67 \mathrm{AU}$.

To obtain the $P_{B}$, we use all MAVEN/MAG magnetic field strength $|B|$ measurements recorded within a cylinder reaching from 120 to $3,000 \mathrm{~km}$ and with a radius of $0.45 R_{M}$ and within the time interval 8 October 2014 to 29 April 2018. The result is presented in Figure 12, which also shows the average $P_{B}$ at low crustal field regions (black line), that is, measurements recorded over regions where the crustal magnetic field strength $\left|B_{c}\right|<20 \mathrm{nT}$ at altitude $400 \mathrm{~km} .\left|B_{c}\right|$ is obtained using the Morschhauser crustal field model (Morschhauser et al., 2014). The decrease in magnetic field strength is visible using a $\left|B_{c}\right|$ limit of $<40 \mathrm{nT}$,

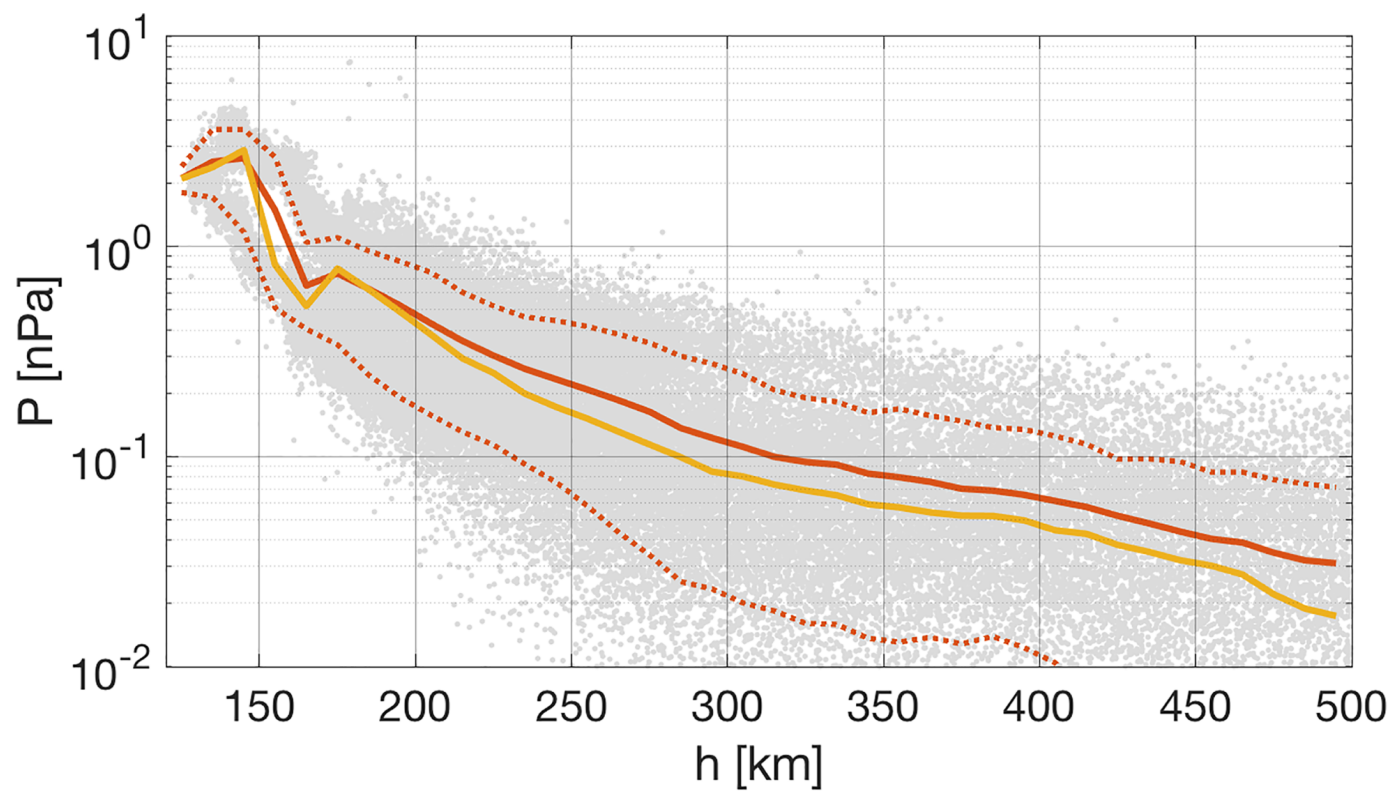

Figure 11. Thermal pressure of the ionosphere (gray dots) estimated from all $n_{e}$ and $T_{e}$ MAVEN measurements recorded during the time interval from 8 October 2014 to 29 April 2018 and within a cylinder centered at the subsolar line, reaching from 120 to $500 \mathrm{~km}$ and with a radius of $0.45 R_{M}$. Presented is also the mean value for each $10-\mathrm{km}$ bin (red), the median (yellow), and the range confining $75 \%$ of the data points for each $10 \mathrm{~km}$ bin (red dotted lines). 


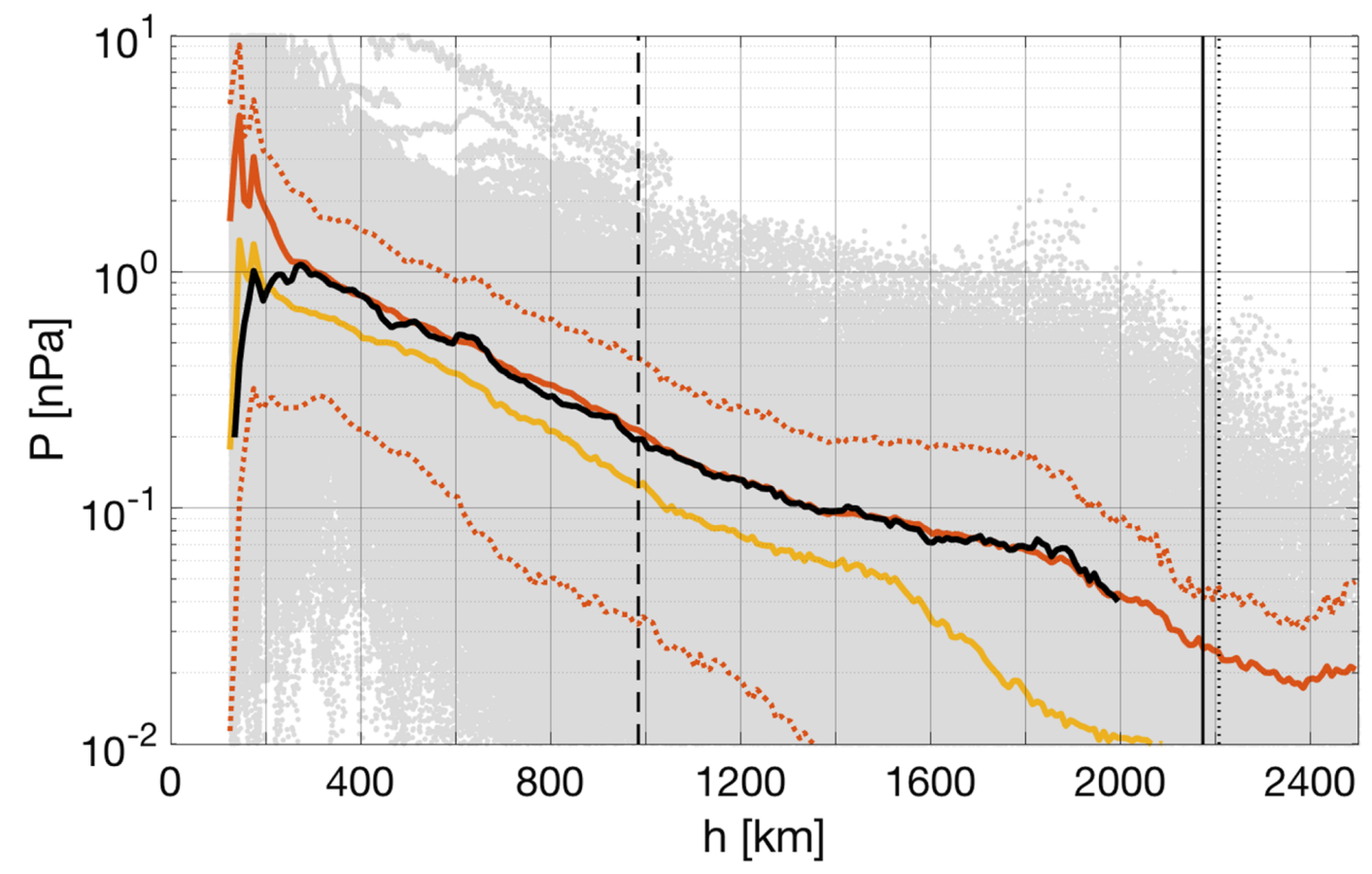

Figure 12. Magnetic pressure (gray dots) estimated from all MAVEN/MAG $|B|$ measurements recorded within a cylinder centered at the subsolar line, reaching from 120 to $3,000 \mathrm{~km}$ and with a radius of $0.45 R_{M}$. Presented is also the mean value (red), the median (yellow), the range confining $75 \%$ of the data points for each $10-\mathrm{km}$ bin (red dotted lines), and the average magnetic pressure for measurements recorded over low crustal field regions; that is, $\left|B_{c}\right|<20 \mathrm{nT}$ at $400 \mathrm{~km}$ (black) estimated from the model by Morschhauser et al. (2014). The vertical black lines mark the MPB (dashed line) and the bow shock (solid line) stand-off distances at 1.29 and $1.64 R_{M}$, given by Vignes et al. (2000). The dotted vertical black line marks the bow shock stand-off distance at $1.65 R_{M}$, given by (Hall et al., 2016).

and for values below $10 \mathrm{nT}$ the number of data points per km bin becomes small enough to affect the statistics. Therefore, the limit was set to $20 \mathrm{nT}$. Figure 12 shows the large difference in magnetic field strength, of more than 1 order of magnitude, between regions with strong (red line) and weak (black line) crustal fields, for altitudes below $\sim 265 \mathrm{~km}$. It is interesting to note the similarities between the average magnetic field strength for weak crustal field regions (black line) with the appearance of the pile-up magnetic field of comets; see, for example, the diamagnetic cavity measurement of comet 67P/Churyumov-Gerasimenko performed by the Rosetta Magnetometer (MAG) and presented by Madanian et al. (2017), their Figures 2 and 4. The Rosetta MAG measurements show much larger field fluctuations at larger distances from the cavity, but the drop at the diamagnetic cavity boundary is similar to the magnetic field drop shown in measurements at low altitudes and over weak crustal field regions at Mars.

The thermal pressure of the magnetosheath is shown in Figure 13, which is estimated from all MAVEN/SWIA $n_{i}$ and $T_{i}$ measurements recorded within the same cylinder shape as used for the $P_{B}$. The data are divided into low quality (light gray) and high quality (dark gray). It is important to point out that the data set presented in Figure 13 is the only data set used in this study that includes measurements that are not of the highest data quality. This exception was made since the highest data quality condition do not provide good statistics for the magnetosheath altitude range. Consequently, the results presented in Figure 13 should be used with care. As discussed earlier, moments from SWIA measurements are computed under the assumption of a $\mathrm{H}^{+}$dominant plasma, meaning that the moments are not correct below the ICB. For that reason, Figure 13 only includes measurements above $650 \mathrm{~km}$, which is an approximate altitude of the upper limit of the ICB according to the results presented in Table 3. Figure 13 also shows the average $P_{t h, m}$ (red), the median $P_{t h, m}$ (yellow), and the range confining $75 \%$ of the data points for each $10-\mathrm{km}$ bin (red dotted lines). The black line gives the average $P_{t h, m}$ measured over low crustal field regions, which are confined to regions where $\left|B_{c}\right|<20 \mathrm{nT}$ at $400 \mathrm{~km}$. Figure 13 shows a very consistent $P_{t h, m}$ over the altitude range $800 \mathrm{~km}$ $\left(1.24 R_{M}\right)$ to $2,065 \mathrm{~km}\left(1.6 R_{M}\right)$, where the average $P_{t h, m}$ only varies between 0.39 and $0.49 \mathrm{nPa}$. 


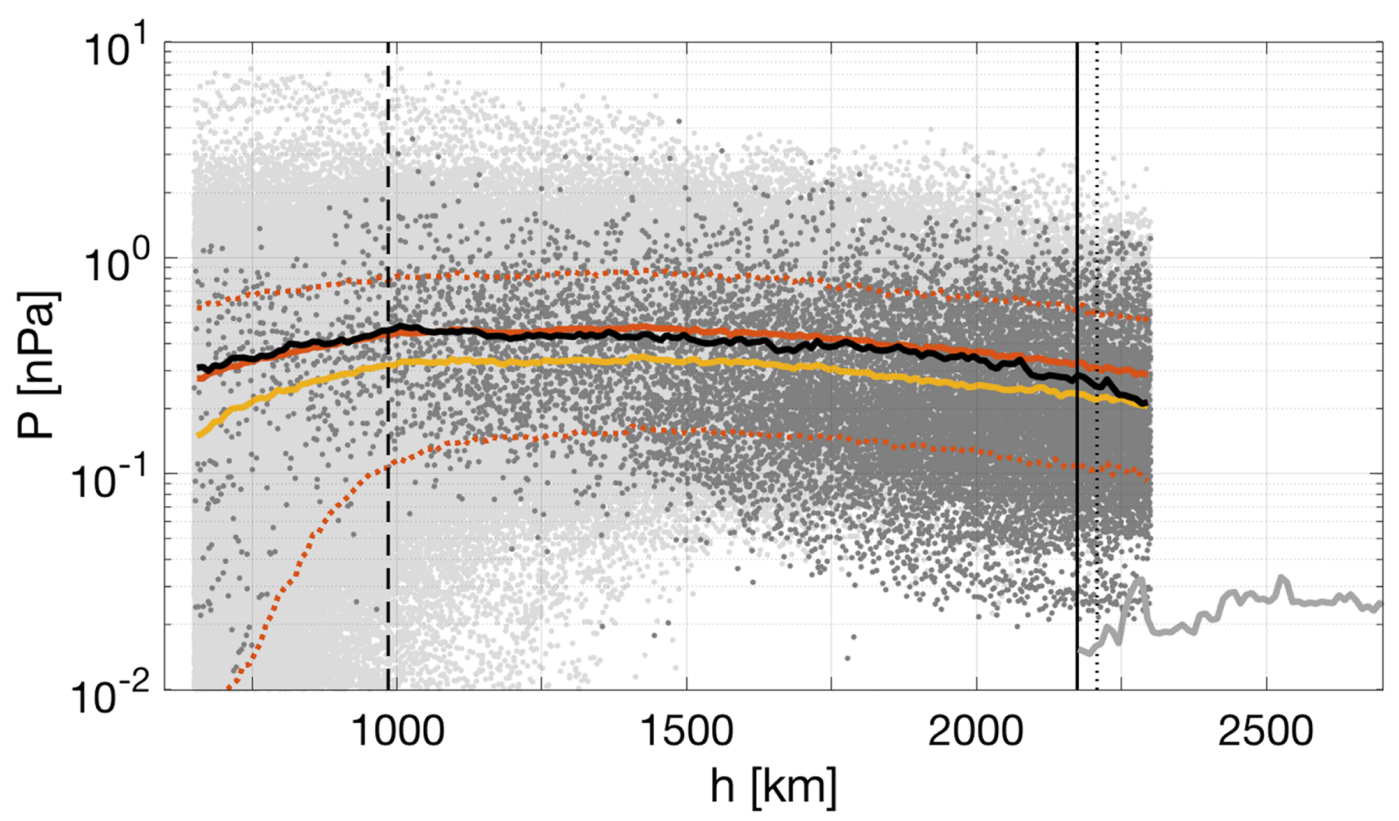

Figure 13. Thermal pressure, all measurements (light gray) and high quality (dark gray), estimated from all MAVEN/SWIA $n_{i}$ and $T_{i}$ measurements recorded within a cylinder centered at the subsolar line, reaching from 650 to $2,300 \mathrm{~km}$ and with a radius of $0.45 R_{M}$. Presented is also the mean value (red), the median (yellow), the range confining $75 \%$ of the data points for each $10-\mathrm{km}$ bin (red dotted lines), and the average magnetic pressure for measurements recorded over low crustal field regions; that is, $|B|<20 \mathrm{nT}$ at $400 \mathrm{~km}$ (black) estimated from the model by Morschhauser et al. (2014). The gray line above $1.64 R_{M}(2,174 \mathrm{~km})$ altitude shows the average thermal pressure estimated from the MAVEN solar wind measurements. The vertical black lines marks the MPB and bow shock stand-off distances as described in Figure 12.

The SWIA data product are provided with two different modes, "Solar Wind Mode" and "Sheath Mode." The different modes are differentiated by which product that was used for the onboard moment calculations. For the Solar Wind Mode the moments are computed from measurements with high energy and angular resolutions but limited energy and angular coverage. For Sheath Mode the moments are computed from measurements that have lower energy and angular resolution but better energy and angular coverage. For a detailed description of the different data products see Halekas et al. (2013). For the data presented in Figure 13 only Sheath Mode was used, in order to show the measured $P_{t h, m}$ in the magnetosheath. In Sheath Mode the ion temperature is commonly overestimated when measuring in the solar wind so Figure 13 does therefore not show the drop in $P_{t h, m}$ that would be expected after the spacecraft crosses the bow shock at an altitude of around $2,200 \mathrm{~km}\left(1.65 R_{M}\right)$. Due to the likely overestimation of the ion temperature in the computations from Sheath Mode measurements in the solar wind, only measurements below 2,300 km $\left(1.68 R_{M}\right)$ are included in Figure 13. Instead, the average $P_{t h}$ using the Solar Wind Mode measurements are shown as the gray line above $1.64 R_{M}(2,174 \mathrm{~km})$, which shows that the thermal pressure commonly would drop with more than one order of magnitude when crossing the bow shock.

For the $P_{d, s w}$ we use all SWIA $n_{i}$ and $v_{i}$ Solar Wind Mode measurements recorded during the time interval 8 October 2014 to 29 April 2018 and within a cylinder centered at SZA $=0^{\circ}$ reaching from 1,000 to 5,500 km and with a radius of $0.9 R_{M}$. The cylinder is illustrated as the outer larger dark gray cylinder in Figure 10. The results are presented in Figure 14, which shows the mean (red) and the median (yellow) values for each $25-\mathrm{km}$ bin. Above 2,000 km the mean pressure values varies from 0.74 to $1.1 \mathrm{nPa}$. Like in Figure 13 the sharp drop in solar wind dynamic pressure that could be expected to be detected after the crossing of the bow shock is absent. However, this is not due to the exclusion of the Sheath Mode measurements but to the relatively large radial ion velocity that is detected also within the expected bow shock distance close to the subsolar point. Relatively large radial ion velocities within the expected bow shock distance were also showed by Halekas et al. (2017), for example, their Figure 6. This shows that for low SZA the dynamic pressure is more prominent than previously expected. This conclusion is also stressed by the results presented in Figure 15, which shows that the dynamic pressure is the dominant pressure term also within the expected bow shock 


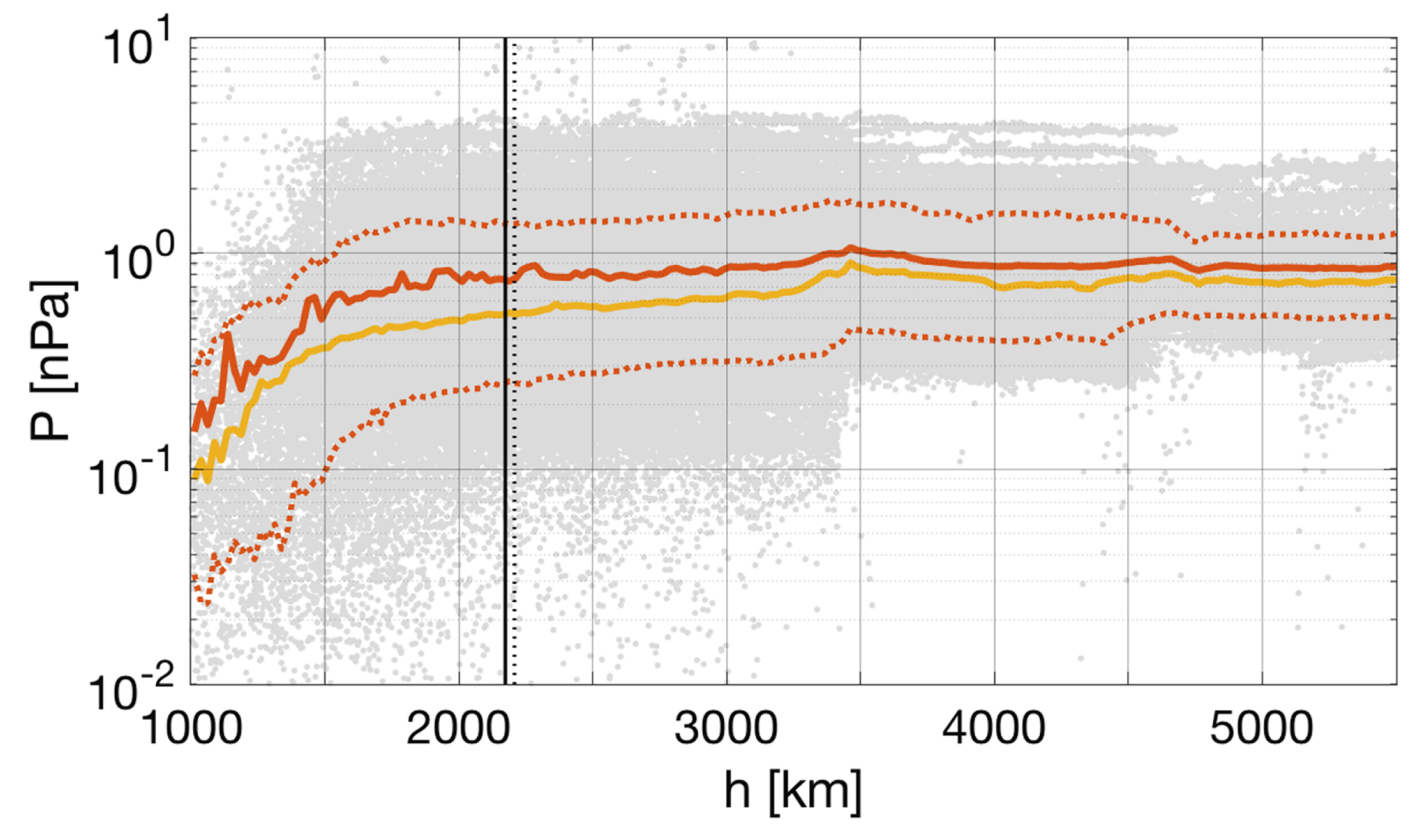

Figure 14. Solar wind dynamic pressure (gray dots) estimated from all MAVEN/SWIA measurements recorded within a cylinder centered at the subsolar line, reaching from 1,000 to $5,500 \mathrm{~km}$ and with a radius of $0.9 R_{M}$. Presented is also the mean value (red), the median (yellow), and the range confining $75 \%$ of the data points for each $25-\mathrm{km}$ bin (red dotted lines). The vertical black lines marks the bow shock stand-off distance as described in Figure 12.

distance, and Figure 16, which shows a gradual decrease of the dynamic pressure instead of the sharp drop that was expected from a pressure balance model.

The statistical results from Figures 11-14 are summarized in Figure 15, which shows the average $P_{t h, i}$ (red), $P_{B}$ (black), $P_{t h, m}$ (purple), and $P_{d, s w}$ (dark gray) from MAVEN measurements recorded on the dayside of the induced magnetosphere. The light gray line shows the total pressure. Due to the lack of $P_{t h, m}$ estimates below 650- and above 2,300-km artificial drops in pressure are found below and above these altitudes. The average for the magnetic pressure (black) is the average for all measurements over weak crustal field regions. As can be seen, based on average pressure values, there are four distinct regions dominated by one pressure term respectively, with a smooth transition between them. As have been shown by the presented orbit segments the transition between the different domains are not always easily identified based on individual events.

We compare the derived average pressure terms with the simulation results from Ma et al. (2004). They used a modified Block-Adaptive Tree Solar Wind Roe-Type Upwind Scheme to solve a four-species set of MHD equations, including the continuity, momentum, magnetic induction, and energy equations, to estimate the different pressure terms along the Sun-Mars line. Figure 16a shows the average thermal pressures $P_{t h, i}($ red) and $P_{t h, m}$ (purple), obtained from the MAVEN measurements, compared to the simulated thermal pressure (green) from Ma et al. (2004). The figure shows slightly lower values of the simulated thermal pressure of the ionosphere (green) when compared to the average (red); however, the simulated $P_{t h, i}$ is within common values for individual orbits. The simulated thermal pressure of the magnetosheath (green) is slightly larger than the average $P_{t h, m}$ (purple), which is related to the slightly weaker magnetic pressure presented in panel (b) (green line). As discussed above the average $P_{t h, m}$ (purple) does not show the drop in pressure that is expected at the bow shock crossing, clearly seen in the simulated $P_{t h, m}$ (green) at the chosen bow shock distance $1.43 R_{M}(1,460 \mathrm{~km})$, since the solar wind measurements are excluded from the estimate and the ion temperature estimated from solar wind measurements using Sheath Mode is incorrect. Figure 16b shows the simulated $P_{B}$ (green) and average $P_{B}$ (black), which agrees well below around $450 \mathrm{~km}\left(1.13 R_{M}\right)$. Figure 16c shows the simulated $P_{d, s w}$ (green) and the average $P_{d, s w}$ (thick gray line), which agrees well except for the sudden drop in simulated dynamic pressure that is seen at the chosen bow shock distance.

The simulated case presented by Ma et al. (2004) is for solar minimum, with weak crustal magnetic field sources, a $3 \mathrm{nT}$ IMF, and a subsolar bow shock distance of $1.43 R_{M}$. These conditions differ slightly from the conditions of the statistical study. The MAVEN measurements used for the statistical study range from 


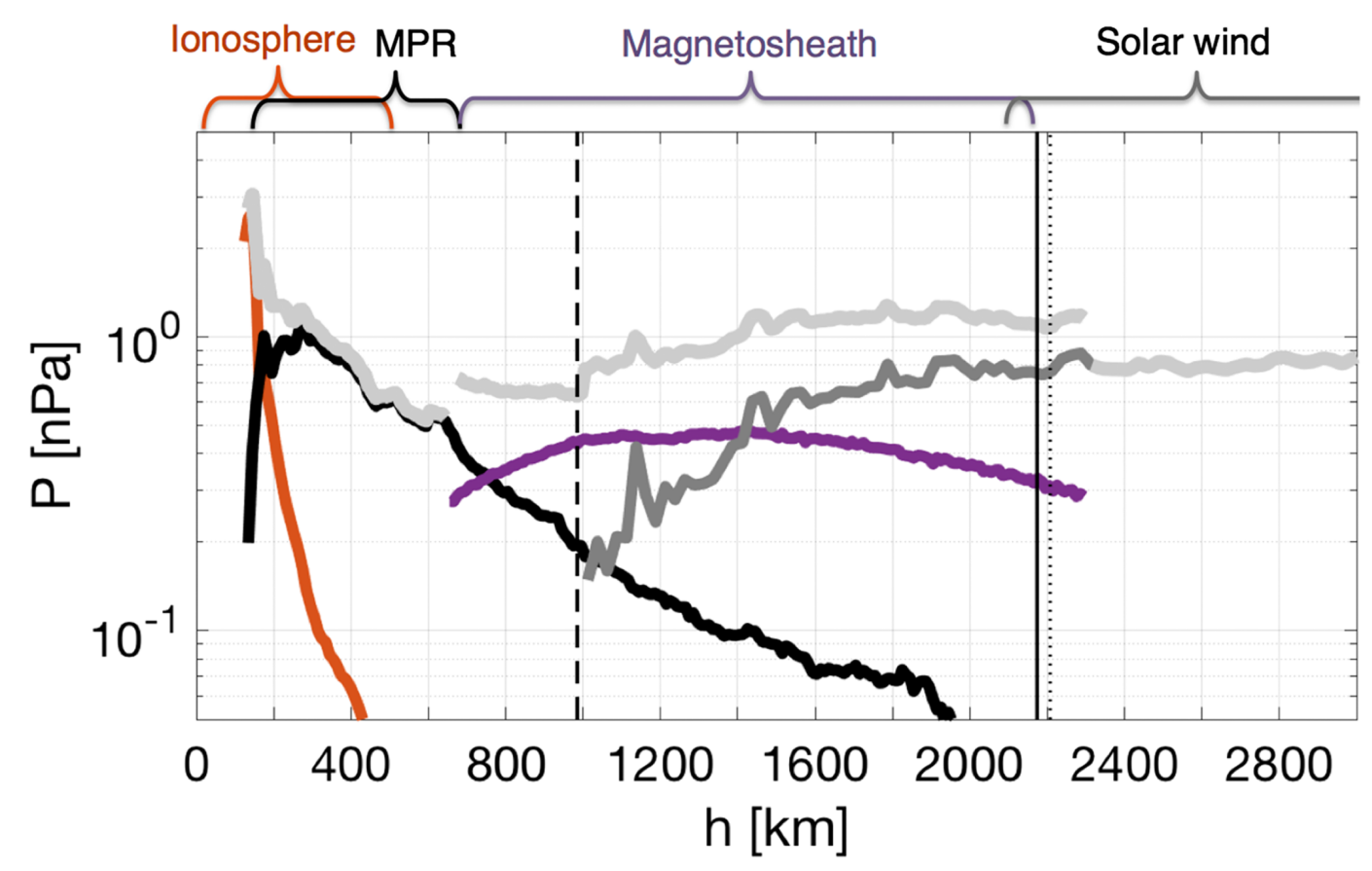

Figure 15. Average $P_{t h, i}$ (red), $P_{B}$ (black), $P_{t h, m}$ (purple), and $P_{d, s w}$ (dark gray) from MAVEN measurements recorded within two cylinder shapes of radius $0.45 R_{M}$ and $0.9 R_{M}$ centered at the subsolar line. The total pressure $P_{\text {tot }}$ is given by the light gray line. The vertical black lines marks the MPB and bow shock stand-off distances as described in Figure 12. MPR = magnetic pile-up region.

8 October 2014 to 29 April 2018 and do therefore cover most of the time period from the solar maximum in 2014 to the solar minimum in 2019. Hence, it is expected that the simulated thermal pressure of the ionosphere would be lower than the measured, which is also seen in Figure 16a. Both the statistical study and the simulation presented in Ma et al. (2004) cover weak crustal field regions. A typical IMF strength of 2 to $3 \mathrm{nT}$ is expected for the statistical study based on the results by Marquette et al. (2018), who used 3 years of MAVEN measurements to obtain the IMF properties at Mars. The bow shock distance of $1.43 R_{M}$ is slightly smaller than what would be expected for the statistical study based on the obtained bow shock stand-off distance of $1.64 R_{M}$ from Vignes et al. (2000) and $1.65 R_{M}$ from Hall et al. (2016). The main reason for the differences between the simulated pressures and the averages of the measured pressures is that the comparison is between averages from years of measurements and a single orbit with a fixed bow shock distance, EUV flux, and IMF strength. Despite these differences, the simulated values agree well with the averages of the measured pressures.

In order to confirm that the 18 orbit segments are a good representation of the system, we compare them with the measurement ranges obtained from the data presented in Figures 11-14. The result is presented in Figure 17. Figure 17a shows the thermal pressure, both $P_{t h, i}$ (red) and $P_{t h, m}$ (purple) of all 18 orbit segments and the thick solid red and purple lines give the ranges confining 75\% of all the measured $P_{t h, i}$ and $P_{t h, m}$ values, for each 10-km bin. The same ranges are also presented as the red dotted lines in Figures 11 and 13. Figure $17 \mathrm{~b}$ shows $P_{B}$ (black) and $P_{d, s w}$ (gray) of all 18 cases, and the thick solid black and gray lines give the ranges confining 75\% of the measured $P_{B}$ and $P_{d, s w}$ values, for each $10 \mathrm{~km}$. The same ranges are presented as the red dotted lines in Figures 12 and 14. Due to the orbit configuration of MAVEN, the MAVEN/SWIA measurements are limited to altitudes below $\sim 6,060 \mathrm{~km}\left(\sim 2.78 R_{M}\right)$. The figure shows that the 18 orbit segments represent the dayside of the Martian induced magnetosphere structure well, except for the solar wind dynamic pressure where the 18 orbit segments commonly are lower than the results from the statistical study. This could be due to that the 18 orbit segments are all for low solar wind dynamic pressure conditions, but it could also be due to that the measurements of the solar wind parameters by MEX/ASPERA-3 gives slightly lower estimations than the measurements from MAVEN/SWIA. To conclude if this is the case, a thorough study of the relation between the MEX/ASPERA-3 and MAVEN/SWIA solar wind measurements is needed, which is beyond the scope of this article. No matter the cause, this does not affect the result 

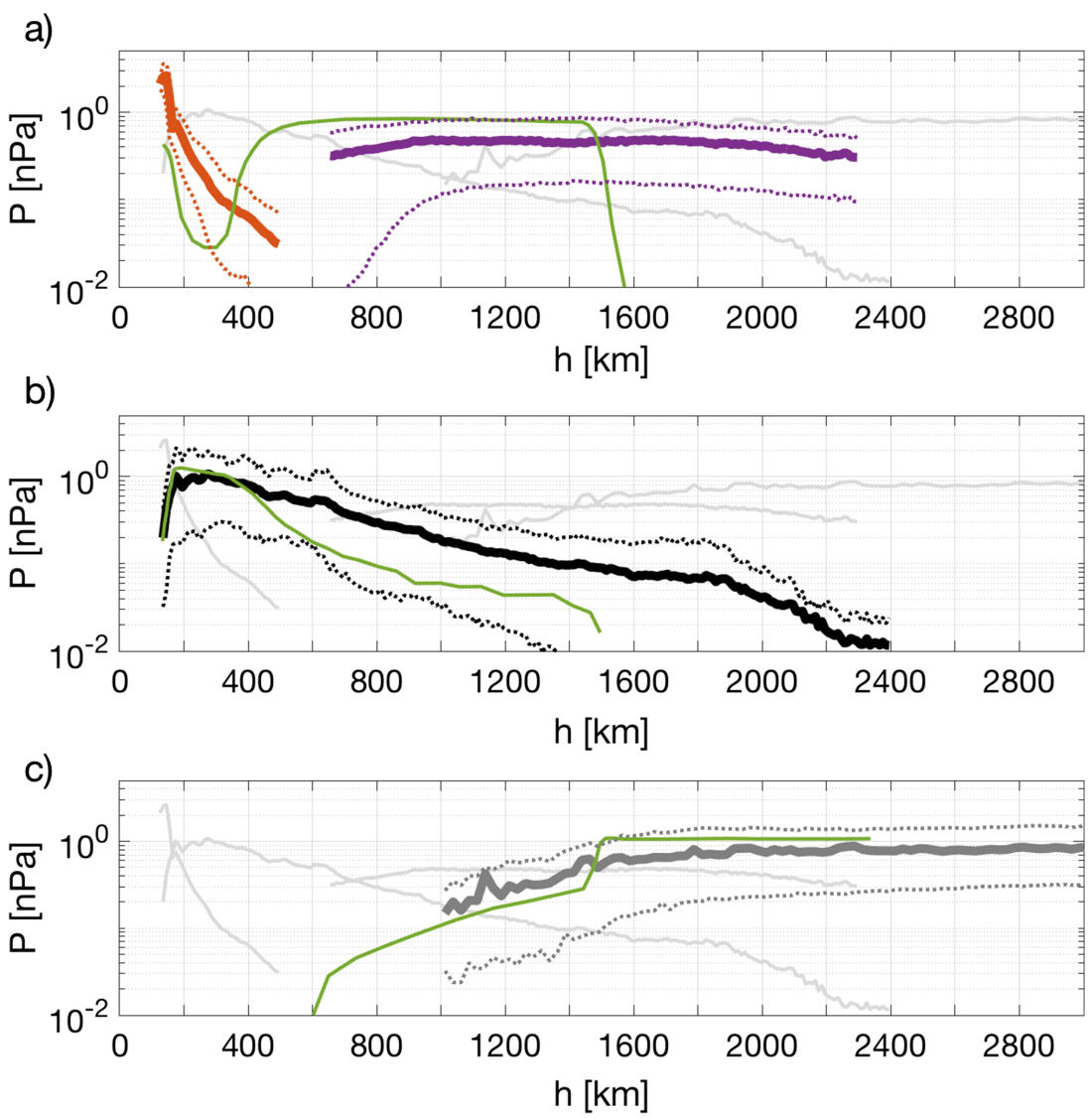

Figure 16. Average $P_{t h, i}$ (red), $P_{B}$ (black), $P_{t h, m}$ (purple), and $P_{d, s w}$ (thick gray line) from MAVEN measurements recorded within two cylinder shapes of radius $0.45 R_{M}$ and $0.9 R_{M}$ centered at the subsolar line, compared to simulation results from Ma et al. (2004; green lines). The panels show the thermal pressure (a), the magnetic pressure (b), and the dynamic pressure (c). The dotted lines give the ranges that confine $75 \%$ of the measured $P_{t h, i}, P_{B}, P_{t h, m}$, and $P_{d, s w}$, which are also presented in Figures 11-14.

presented in this study since the MEX/ASPERA-3 solar wind measurements have been used only to exclude high $P_{d, s w}$ events and to obtain the magnitude of the $P_{d, s w}$, and not to estimate any of the boundary altitudes.

Figure 17c shows all 18 orbit segments and the medians of $P_{t h, i}$ (red), $P_{B}$ (black), $P_{t h, m}$ (purple) obtained from all measurements presented in Figures 11-13. Included are also the median altitudes of the boundaries listed in Table 3, the upper and lower pressure balance boundaries (shaded regions), PEB, ICB, and IMB. Please note that the upper boundary of the PEB, at $630 \mathrm{~km}$, and the lower boundary of the IMB, at $647 \mathrm{~km}$, are found within the boundaries of the ICB, at 602 and $657 \mathrm{~km}$.

A cartoon showing the various boundaries and some of the characteristics of the dayside of the induced magnetosphere of Mars is shown in Figure 18. The figure includes the upper and lower pressure balance boundary, the PEB, ICB, IMB, and the ion/electron and magnetic field characteristics of the various regions.

\section{Discussion and Conclusions}

From the data presented in this study we can confirm that the dayside of the Martian magnetosphere has two distinctly different regions: the magnetosheath and the ionosphere. These two regions are clearly separated in all of the studied data, in terms of their respective environment properties. On the contrary, the physical separation between the regions and its characterization is complex. The two regions are separated by a transition region that includes a change in magnetic field strength and fluctuation, dominant ion species, and electron and ion energies and densities. Traditionally, many names have been used for the transition region: the ICB, the IMB, the MPB, etc., but they do not seem to have fulfilled the need for categorizing this region since the discussion is still ongoing. This is mainly due to the fact that the above boundaries commonly are 
a)
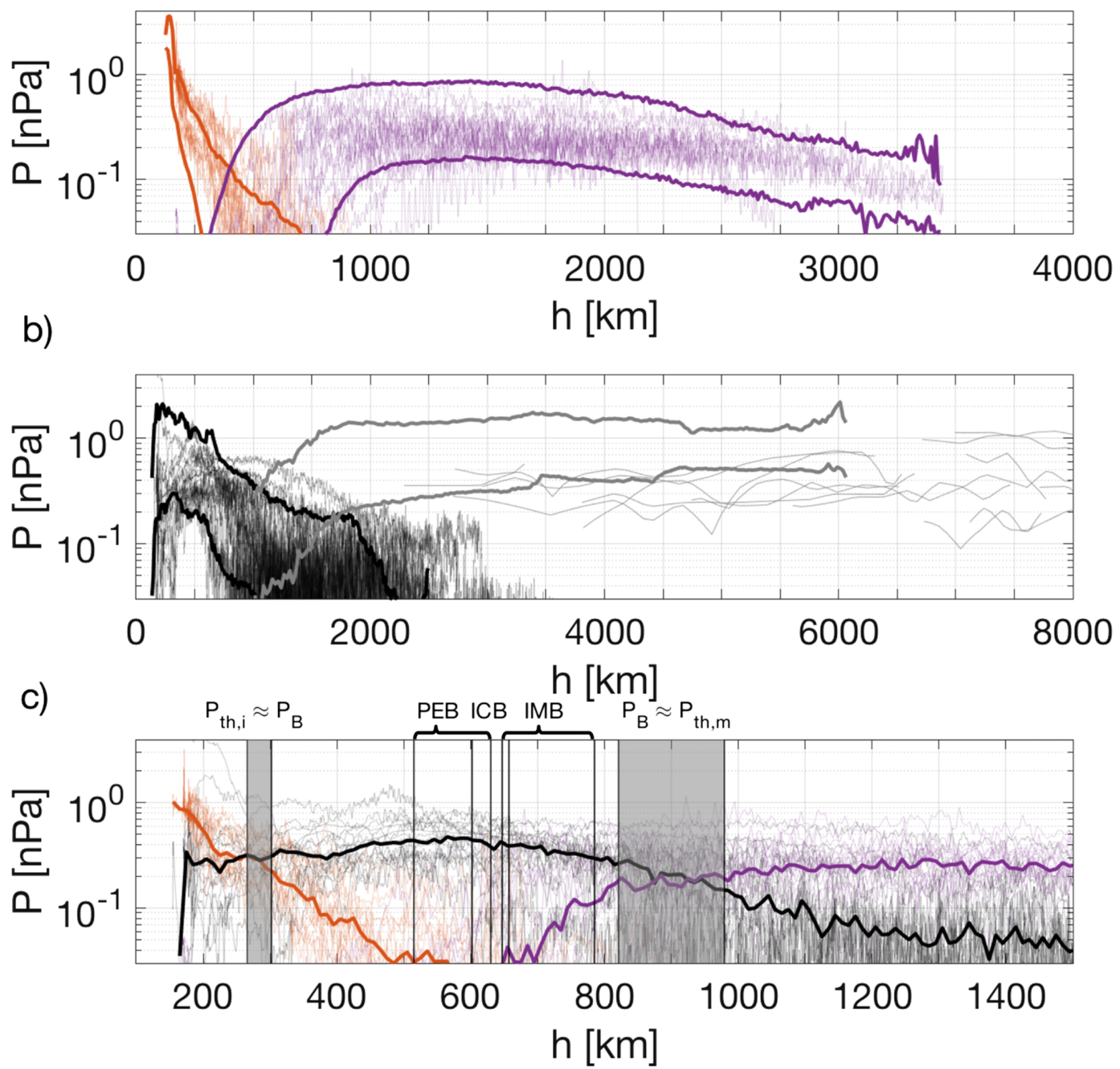

Figure 17. Panel (a) shows the $P_{t h, i}$ (red) and $P_{t h, m}$ (purple) from all the 18 orbit segments, listed in Table 2, compared to the ranges confining $75 \%$ of all the $P_{t h, i}$ (thick red lines) estimates presented in Figure 11 and the $P_{t h, m}$ (thick purple lines) estimates presented in Figure 13, for altitudes up to 4,000 km. Panel (b) shows the $P_{B}$ (black) and $P_{d, s w}$ (gray) from all the 18 orbit segments compared to the range confining $75 \%$ of all the $P_{B}$ (thick black lines) estimates presented in Figure 12 and the $P_{d, s w}$ (thick gray lines) estimates presented in Figure 14, for altitudes up to 8,000 km. The ranges are given for each 10-km bin. Panel (c) shows all 18 orbit segments and the median values (thick red, black, and purple lines) derived from the $P_{t h, i}, P_{B}, P_{t h, m}$ estimates presented in Figures 11-14. Panel (c) also shows the median altitudes (vertical black lines) of the upper and lower pressure balance boundaries (shaded areas), the photoelectron boundary (PEB), ion composition boundary (ICB), and induced magnetosphere boundary (IMB), listed in Table 3.

defined by measurements from only one or two instruments, which is partly due to that no mission before MAVEN held an equally comprehensive plasma package. The MAVEN mission has finally made it possible to compare the different boundaries and such a comparison is presented in this article. We can conclude that the transition region, at SZAs $<60^{\circ}$, is neither a sharp nor easily defined boundary.

When comparing the different boundaries included in this study, we see that any future study focusing on the magnetosheath should benefit from using the conservative estimate of the lower boundary of the magnetosheath: the pressure balance boundary where $P_{B} \approx P_{t h, m}$. Above the $P_{B} \approx P_{t h, m}$ boundary the plasma and magnetic field properties are all magnetosheath-like. This can be seen in Figures 3, 6, and 9. Finding an estimated altitude for the upper limit of the ionosphere is more complex since the lower pressure balance boundary, where $P_{t h, i} \approx P_{B}$, often is located well within the ionosphere and is therefore not recommended to be used as an upper boundary of the ionosphere. This is concluded from studying the energy spectra of ions 


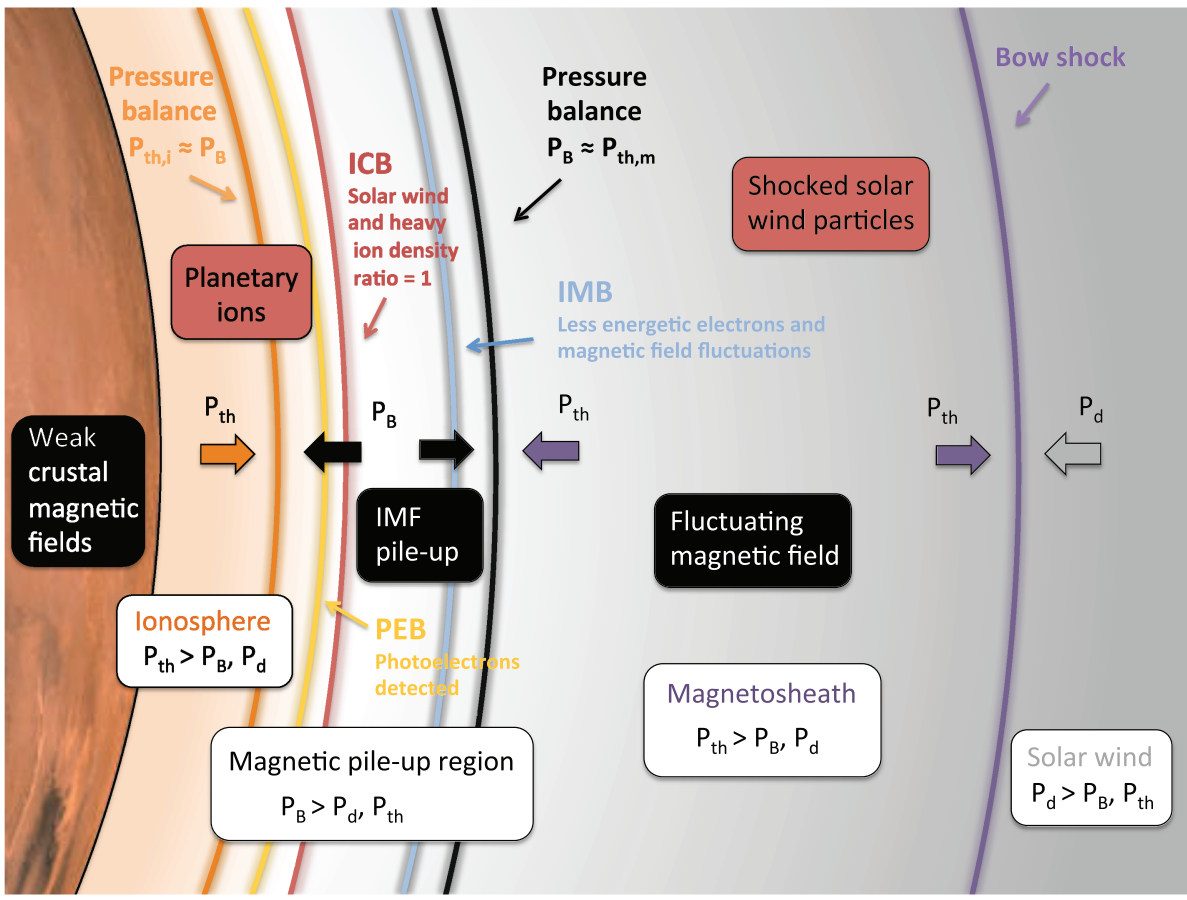

Figure 18. Overview of the various boundaries and regions located on the dayside of the Martian induced magnetosphere. The figure illustrates the magnetospheric structure during average or low solar wind dynamic pressure and for weak crustal field regions. The various regions and their dominating pressure terms are stated in the white text fields. The lines illustrating the various boundaries give approximate boundary altitudes, but they do not correspond to the actual boundary thicknesses. In this study the notation magnetic pile-up region is used for the region where the magnetic pressure dominates over the thermal pressure, which gives it an extension from the lower pressure balance boundary where $P_{t h, i} \approx P_{B}$ (orange boundary) to the upper pressure balance boundary where $P_{B} \approx P_{t h, m}$ (black boundary). This means that both the magnetosheath and the ionosphere overlap with the magnetic pile-up region. The figure also shows the changes in the magnetic field (black text fields) and in ion/electron characteristics (red text fields). ICB = ion composition boundary; IMF = interplanetary magnetic field; IMB = induced magnetosphere boundary; $\mathrm{PEB}=$ photoelectron boundary.

and electrons as well as magnetic field fluctuations and dominant ion species. Often, the clear ionosphere appearance seems to be located somewhere below the PEB, but none of the studied boundaries seems to clearly define the upper boundary of the ionosphere as well as the $P_{B} \approx P_{t h, m}$ boundary defines the lower boundary of the magnetosheath.

We also show that none of the other boundaries, that is, the PEB, ICB, and IMB, define the transition region between the ionosphere and the magnetosheath of Mars. They only suffice to identify a position at some location within the transition region. For example, the ICB is usually defined as the region where there are equal number of solar wind ions and ions of ionospheric origin or more precisely a choice of some dominant ionospheric ion species. This simply means that the measurements are performed within a region of mixing between the solar wind and the Martian ionosphere, but this is also correct for a certain range where the solar wind to heavy ion ratio is larger or smaller than 1 . It is possible that using some ratio range instead of a single value would increase the ICB width to eventually include most of the transition region. However, the needed ratio range cannot be obtained without also studying the energy spectra of ions and electrons as well as the magnetic field fluctuations. Thus, the PEB, ICB, and IMB do not define the transition region between the magnetosheath and the ionosphere, but they can be used to indicate an approximate altitude within the transition region. This can also be seen in Figures 3, 6, and 9.

It might be tempting to refer to the transition region as the MPR since this region is roughly located between the ionosphere and the magnetosheath. This region has an increased magnetic field strength, due to the pile up of the IMF, and since this is the region where the thermal pressure of both the ionosphere and the magnetosheath are small, the magnetic pressure is the dominant pressure term. However, the lower part of the MPR is often well within the upper part of the ionosphere and is therefore not the same as the transition 
region. A more accurate name would be the induced magnetosphere boundary but with a wider definition than previously used, including the definition used in this study. A correctly defined IMB should include changes in magnetic field strength and fluctuations, dominant ion species, electron and ion densities and energy distributions.

It is also interesting to note that when using the previously defined conditions to detect the PEB, ICB, and IMB, they frequently overlap and their relative distances and order of appearance changes from orbit to orbit. Their behavior points to the conclusion that the Martian magnetosphere is very dynamic, which is also clearly shown in the large spread of the pressure values presented in Figures 11-14, to the extent that finding the quasi-static situation might even be the exception and not the norm. This would also be true when the variability of the solar wind is of timescales shorter than the time required for the Martian system to reach equilibrium.

Another interesting feature detected in the presented data is the double boundary crossings, which is yet another indicator that the Martian dayside magnetosphere is a very dynamic region. Both in the sense that the magnetospheric structure changes from orbit to orbit but also that changes in the structure might occur so rapidly that the spacecraft encounters double boundary crossings, as shown in Figures 2 and 3. These are commonly found in MAVEN measurements and have unfortunately been mainly ignored in previous studies. A detailed study of the characteristics of double boundary crossings would be an interesting way to reveal more about the dynamics of the Martian magnetosphere and to better understand the physical processes that creates the Martian magnetospheric structure.

Despite the structural changes, the same pressure regions appeared in all of our studied orbits: the thermal pressure of the ionosphere $P_{t h, i}$, the magnetic pressure of the MPR $P_{B}$, and the thermal pressure of the magnetosheath $P_{t h, m}$. We did not encounter an orbit where the $P_{B}$ was so small or $P_{t h, m}$ so large that $P_{B}$ never was the dominant term. However, studying the piled-up magnetic field and its relative strength and dependence on the varying solar wind would be an interesting future study that would reveal more about the induced magnetosphere of Mars.

We obtained an average ionospheric thermal pressure of maximum $2.9 \mathrm{nPa}$ at $145 \mathrm{~km}$ and decreasing to $0.04 \mathrm{nPa}$ at $450 \mathrm{~km}$, see Figure 11, and an average magnetic pressure varying between 0.6 and $1 \mathrm{nPa}$ between 155 and $500 \mathrm{~km}$ (for the low crustal field measurements), see Figure 12. The average thermal pressure of the magnetosheath is varying between 0.4 and $0.5 \mathrm{nPa}$ between 800 and 2,065 km, see Figure 13, and the average solar wind dynamic pressure is varying between 0.7 and $1.1 \mathrm{nPa}$ above 2,000 km, see Figure 14 . These estimates are in agreement with earlier simulations of pressure terms during solar minimum and over weak crustal magnetic field sources, see Figure 16.

The results presented in this study show that the transition region between the Martian dayside magnetosheath and ionosphere, that is, the IMB, needs further investigation. In addition, future studies of the Martian IMB should be multi-instrumental in order to correctly characterize this boundary and its defining properties, which are changes in magnetic field strength and fluctuations, dominant ion species, electron and ion densities and energy distributions.

\section{Acknowledgments}

The authors would like to thank Elena Budnik from the CDPP/AMDA team for providing the MEX data and for helpful discussions about the data quality. The MAVEN project is supported by NASA through the Mars Exploration Program. The MAVEN/SWEA instrument is also supported by the French National Centre for Space Studies (CNES). MAVEN and MEX data are available through the Planetary Plasma Interactions node of NASA's Planetary Data System (https://pds-ppi.igpp. ucla.edu/). Data analysis was partly performed with the AMDA science analysis system provided by the CDPP and supported by CNRS, CNES, Paris Observatory, and Paul Sabatier University, Toulouse.

\section{References}

Acuña, M. H., Connerney, J. E. P., Wasilewski, P., Lin, R. P., Anderson, K. A., Carlson, C. W., et al. (1998). Magnetic field and plasma observations at Mars: Initial results of the Mars Global Surveyor mission. Science, 279(5357), 1676-1680.

Andersson, L., Ergun, R. E., Delory, G. T., Eriksson, A., Westfall, J., Reed, H., et al. (2015). The Langmuir Probe and Waves (LPW) instrument for MAVEN. Space Science Reviews, 195, 173-198. https://doi.org/10.1007/s11214-015-0194-3

Barabash, S., Lundin, R., Andersson, H., Brinkfeldt, K., Grigoriev, A., Gunell, H., et al. (2006). The Analyzer of Space Plasmas and Energetic Atoms (ASPERA-3) for the Mars Express mission. Space Science Reviews, 126, 113-164. https://doi.org/10.1007/s11214-006-9124-8

Benna, M., Mahaffy, P. R., Grebowsky, J. M., Fox, J. L., Yelle, R. V., \& Jakosky, B. M. (2015). First measurements of composition and dynamics of the Martian ionosphere by MAVEN's Neutral Gas and Ion Mass Spectrometer. Geophysical Research Letter, 42, 8958-8965. https://doi.org/10.1002/2015GL066146

Bertucci, C., Mazelle, C., Crider, D. H., Vignes, D., Acuña, M. H., Mitchell, D. L., et al. (2003). Magnetic field draping enhancement at the Martian magnetic pileup boundary from Mars Global Surveyor observations. Geophysical Research Letters, 30(2), 1099. https://doi.org/ 10.1029/2002GL015713

Bößwetter, A., Bagdonat, T., Motschmann, U., \& Sauer K. (2004). Plasma boundaries at Mars: A 3-D simulation study. Annals of Geophysics, 22, 4363-4379. https://doi.org/10.5194/angeo-22-4363-2004

Breus, T. K., Krymskii, A. M., Lundin, R., Dubinin, E. M., Luhmann, J. G., Yeroshenko, Y. G., et al. (1991). The solar wind interaction with Mars: Consideration of Phobos 2 mission observations of an ion composition boundary on the dayside. Journal of Geophysical Research, 96(A7), 11,165-11,174. 
Chicarro, A., Martin, P., \& Trautner, R. (2004). The Mars Express mission: An overview in Mars Express: The scientific payload Edited by Wilson, A., \& Chicarro, A., vol. 1240, pp. 3-13: ESA Special Publication.

Connerney, J. E. P., Espley, J., Lawton, P., Murphy, S., Odom, J., Oliversen, R., \& Sheppard, D. (2015). The MAVEN magnetic field investigation. Space Science Reviews, 195, 257-291. https://doi.org/10.1007/s11214-015-0169-4

Crider, D., Cloutier, P., Law, C., Walker, P., Chen, Y., Acuña, M., et al. (2000). Evidence of electron impact ionization in the magnetic pileup boundary of Mars. Geophysical Research Letters, 27(1), 45-48.

Dubinin, E., Fraenz, M., Woch, J., Chanteur, G., Duru, F., Akalin, F., et al. (2008). Plasma environment of Mars as observed by simultaneous MEX-ASPERA-3 and MEX-MARSIS observations. Geophysical Research Letters, 113, A10217. https://doi.org/10.1029/2008JA013355

Dubinin, E., Modolo, R., Fraenz, M., Woch, J., Duru, F., Akalin, F., et al. (2008). Structure and dynamics of the solar wind/ionosphere interface on Mars: MEX-ASPERA-3 and MEX-MARSIS observations. Geophysical Research Letters, 35, L11103. https://doi.org/10.1029/ 2008GL033730

Edberg, N. J. T., Brain, D. A., Lester, M., Cowley, S. W. H., Modolo, R., Fränz, M., \& Barabash, S. (2009). Plasma boundary variability at Mars as observed by Mars Global Surveyor and Mars Express. Annals of Geophysics, 27, 3537-3550. https://doi.org/10.5194/ angeo-27-3537-2009

Espley, J. R. (2018). The Martian magnetosphere: Areas of unsettled terminology. Journal of Geophysical Research: Space Physics, 123, 4521-4525. https://doi.org/10.1029/2018JA025278

Garnier, P., Steckiewicz, M., Mazelle, C., Xu, S., Mitchell, D., Holmberg, M. K. G., et al. (2017). The Martian photoelectron boundary as seen by MAVEN. Journal of Geophysical Research: Space Physics, 122, 10,472-10,485. https://doi.org/10.1002/2017JA024497

Grard, R., Pedersen, A., Klimov, S., Savin, S., Skalsky, A., \& Kennel, C. (1989). First measurements of plasma waves near Mars. Nature, 341, 607-609.

Halekas, J. S., Brain, D. A., Ruhunusiri, S., McFadden, J. P., Mitchell, D. L., Mazelle, C., et al. (2016). Plasma clouds and snowplows: Bulk plasma escape from Mars observed by MAVEN. Geophysical Research Letters, 43, 1426-1434. https://doi.org/10.1002/2016GL067752

Halekas, J. S., McFadden, J. P., Brain, D. A., Luhmann, J. G., DiBraccio, G. A., Connerney, J. E. P., et al. (2018). Structure and variability of the Martian ion composition boundary layer. Journal of Geophysical Research: Space Physics, 123, 8439-8458. https://doi.org/10.1029/ 2018JA025866

Halekas, J. S., Ruhunusiri, S., Harada, Y., Collinson, G., Mitchell, D. L., Mazelle, C., et al. (2017). Structure, dynamics, and seasonal variability of the Mars-solar wind interaction: MAVEN Solar Wind Ion Analyzer in-flight performance and science results. Journal of Geophysical Research: Space Physics, 122, 547-578. https://doi.org/10.1002/2016JA023167

Halekas, J. S., Taylor, E. R., Dalton, G., Johnson, G., Curtis, D. W., McFadden, J. P., et al. (2013). The solar wind ion analyzer for MAVEN. Space Science Reviews, 195, 125-151.

Hall, B. E. S., Lester, M., Sánchez-Cano, B., Nichols, J. D., Andrews, D. J., Edberg, N. J. T., et al. (2016). Annual variations in the Martian bow shock location as observed by the Mars Express mission. Journal of Geophysical Research: Space Physics, 121, 11,474-11,494. https:// doi.org/10.1002/2016JA023316

Jakosky, B. M., Lin, R. P., Grebowsky, J. M., Luhmann, J. G., Mitchell, D. F., Beutelschies, G., et al. (2015). The Mars Atmosphere and Volatile Evolution (MAVEN) Mission. Space Science Reviews, 195, 3-48.

Lundin, R., Barabash, S., Andersson, H., Holmström, M., Grigoriev, A., Yamauchi, M., et al. (2004). Solar wind-induced atmospheric erosion at Mars: First results from ASPERA-3 on Mars Express. Science, 305, 1933.

Lundin, R., Zakharov, A., Pellinen, R., Borg, H., Hultqvist, B., Pissarenko, N., et al. (1989). First measurements of the ionospheric plasma escape from Mars. Nature, 341, 609-612.

Ma, Y. J., Nagy, A. F., Sokolov, I. V., \& Hansen, K. C. (2004). Three-dimensional, multispecies, high spatial resolution MHD studies of the solar wind interaction with Mars. Journal of Geophysical Research, 109, A07211. https://doi.org/10.1029/2003JA010367

Madanian, H., Cravens, T. E., Burch, J., Goldstein, R., Rubin, M., Nemeth, Z., et al. (2017). Plasma environment around comet 67P/Churyumov-Gerasimenko at perihelion: Model comparison with Rosetta data. The Astronomical Journal, 153(30).

Mantas, G. P., \& Hanson, W. B. (1979). Photoelectron fluxes in the Martian ionosphere. Journal of Geophysical Research, 84(A2), 369-385.

Marquette, M., Lillis, R. J., Halekas, J. S., Luhmann, J. G., Gruesbeck, J. R., \& Espley, J. R. (2018). Autocorrelation study of solar wind plasma and IMF properties as measured by the MAVEN spacecraft. Journal of Geophysical Research: Space Physics, 123, 2493-2512. https://doi. org/10.1002/2018JA025209

Matsunaga, K., Seki, K., Brain, D. A., Hara, T., Masunaga, K., McFadden, J. P., et al. (2017). Statistical study of relations between the induced magnetosphere, ion composition, and pressure balance boundaries around Mars based on MAVEN observations. Journal of Geophysical Research: Space Physics, 122, 9723-9737. https://doi.org/10.1002/2017ja024217

Mazelle, C., Winterhalter, D., Sauer, K., Trotignon, J. G., Acuña, M. H., Baumgärtel, K., et al. (2004). Bow shock and upstream phenomena at Mars. Space Science Reviews, 111, 115-181.

McFadden, J. P., Kortmann, O., Curtis, D., Dalton, G., Johnson, G., Abiad, R., et al. (2015). MAVEN SupraThermal and Thermal Ion Composition (STATIC) instrument. Space Science Reviews, 195, 199-256.

Mitchell, D. L., Lin, R. P., Rème, H., Crider, D. H., Cloutier, P. A., Connerney, J. E. P., et al. (2000). Oxygen Auger electrons observed in Mars' ionosphere. Geophysical Research Letters, 27(13), 1871-1874.

Mitchell, D. L., Mazelle, C., Sauvaud, J.-A., Thocaven, J.-J., Rouzaud, J., Fedorov, A., et al. (2016). The MAVEN Solar Wind Electron Analyzer. Space Science Reviews, 200, 495-528.

Morschhauser, A., Lesur, V., \& Grott, M. (2014). A spherical harmonic model of the lithospheric magnetic field of Mars. Journal of Geophysical Research: Planets, 119, 1162-1188. https://doi.org/10.1002/2013JE004555

Nagy, A. F., Winterhalter, D., Sauer, K., Cravens, T. E., Brecht, S., Mazelle, C., et al. (2004). The plasma environment of Mars. Space Science Reviews, 111(1-2), 33-114.

Riedler, W., Möhlmann, D., Oraevsky, V. N., Schwingenschuh, K., Yeroshenko, Ye., Rustenbach, J., et al. (1989). Magnetic fields near Mars: First results. Nature, 341, 604-607.

Rosenbauer, H., Shutte, N., Apáthy, I., Galeev, A., Gringauz, K., Grünwaldt, H., et al. (1989). Ions of martian origin and plasma sheet in the Martian magnetosphere: Initial results of the TAUS experiment. Nature, 341, 612-614.

Russell, C. T. (Ed.) (2007). The plasma environment of Mars Edited by Russell, C. T.Dordrecht, The Netherlands: Springer.

Shutte, N. M., Király, P., Cravens, T. E., Dyachkov, A. V., Gombos, T. I., Gringuaz, K. I., et al. (1989). Observation of electron and ion fluxes in the vicinity of Mars with the HARP spectrometer. Nature, 341, 614-616.

Trotignon, J. G., Mazelle, C., Bertucci, C., \& Acuña, M. H. (2006). Martian shock and magnetic pile-up boundary positions and shapes determined from the Phobos 2 and Mars Global Surveyor data sets. Planetary and Space Science, 54(4), 357-369. 
Vignes, D., Mazelle, C., Rme, C., Acuña, M. H., Connerney, J. E. P., Lin, R. P., et al. (2000). The solar wind interaction with Mars: Locations and shapes of the bow shock and the magnetic pile-up boundary from the observations of the MAG/ER Experiment onboard Mars Global Surveyor. Geophysical Research Letters, 27(1), 49-52. https://doi.org/10.1029/1999GL010703

Xu, S., Liemohn, M. W., Dong, C., Mitchell, D. L., Bougher, S. W., \& Ma, Y. (2016). Pressure and ion composition boundaries at Mars. Journal of Geophysical Research: Space Physics, 121, 6417-6429. https://doi.org/10.1002/2016JA022644

\section{Erratum}

In the originally published version of this papers, updated images for Figures 12, 13, 14, 15, 16 and 17 showing addition tick marks and grids were not used. These errors have since been corrected and this version may be considered the authoritative version of record. 